\title{
Determining Evolutionary Spectra from non-Stationary Autocorrelation Functions
}

\author{
Brett A. Benowitz ${ }^{1}$, Michael D. Shields ${ }^{2}, \&$ George Deodatis ${ }^{3}$ \\ ${ }^{1}$ Applied Sciences Division, Weidlinger Associates, Inc. \\ 2 Dept. of Civil Eng., Johns Hopkins University \\ ${ }^{3}$ Dept. of Civil Eng. \& Eng. Mechanics, Columbia University
}

\begin{abstract}
For non-stationary stochastic processes, the classic integral expression for computing the autocorrelation function from the evolutionary power spectral density (evolutionary spectrum) developed by Priestley is not invertible in a unique way. Thus, the evolutionary spectrum cannot be determined analytically from a given autocorrelation function. However, the benefits of an efficient inversion from autocorrelation to evolutionary spectrum are vast. In particular, it is more straightforward to estimate the autocorrelation function from measured data, yet efficient simulation depends on knowing the evolutionary spectrum. This work examines the existence and uniqueness of such an inversion from the autocorrelation to the evolutionary spectrum under a certain set of conditions. It is established that uniqueness of the inversion is likely although it is not proven. A methodology is presented to determine the evolutionary spectrum from a prescribed or measured non-stationary autocorrelation function by posing the inversion as a discrete optimization problem. This method demonstrates the ability to perform the inversion but is computationally very expensive. An improved method is then proposed to enhance the computational efficiency and is compared with some established optimization methods. Numerical examples are provided throughout to demonstrate the capabilities of the proposed methodologies.
\end{abstract}

Keywords: stochastic process; stochastic field; evolutionary spectrum; non-stationary; non-homogeneous 


\section{Introduction \& motivation}

Through increases in both the computational power and efficiency of algorithms available to scientists and engineers, it is becoming increasingly possible to consider problems in a fully stochastic framework that were once only approachable deterministically. Monte Carlo simulation remains at the forefront of the analysis methods for its robustness. In fact, for many classes of problems (and several of practical interest), Monte Carlo simulation is the only option to fully characterize the system. This may be true, for example, in evaluating the structural response to seismic ground motion; characterizing the morphology of statistically non-homogeneous random media - like Functionally Graded Materials (FGMs), evaluating the response of bridges to wind loads; and is particularly true when strong nonlinearities are present.

The bedrock of Monte Carlo methods is the efficient and accurate simulation of sample realizations. For the examples previously listed, this translates to generating sample earthquake time histories, material morphologies, and wind velocity time histories/fields, respectively. The Spectral Representation Method (SRM) [1] is widely used for the simulation of sample realizations of Gaussian stochastic processes and recent developments have widely enhanced its applicability for non-Gaussian processes $[2,3,4]$ by coupling with Grigoriu's translation process theory [5]. SRM is the method employed in this work. However, there are many other simulation techniques available (e.g. Autoregressive Moving Average (ARMA) models $[6,7,8,9,10,11]$ and the Karhunen-Loève expansion [12, 13, 14, 15], among others) that will not be discussed in detail in this work. The SRM, in particular, is commonly used in the fields of civil engineering and applied mechanics, due partly to its nice physical interpretation afforded by the trigonometric basis functions that give the power spectral density function units of power at different frequencies. Additionally, it is comparatively mathematically simple and numerically easy to implement, as the simulated samples are generated by a finite sum of cosine functions or fast Fourier transform.

This work is specifically concerned with non-stationary stochastic processes ${ }^{1}$. A process

\footnotetext{
${ }^{1}$ The terms "non-stationary" and "non-homogeneous" are used interchangeably throughout this paper,
} 


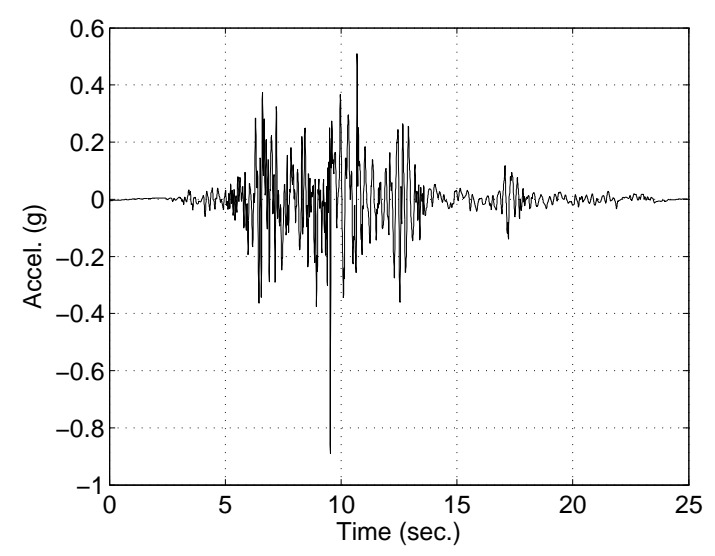

(a) Ground acceleration
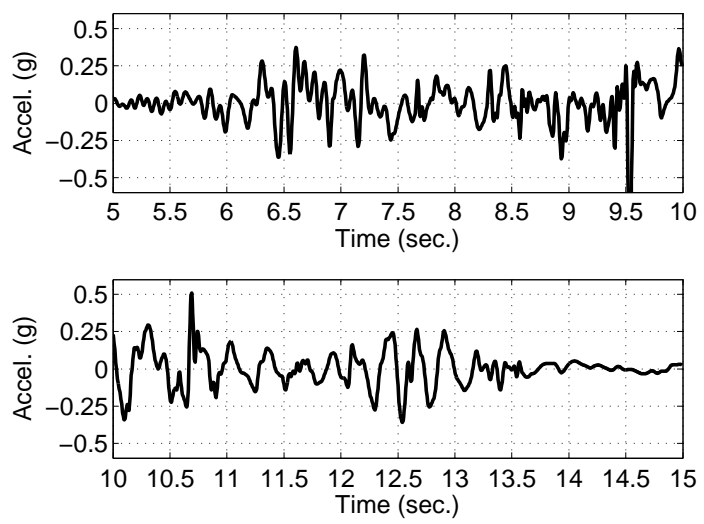

(b) Zoom details on ground acceleration.

Figure 1: Example seismic ground acceleration: 1989 Loma Prieta earthquake. Data source: Pacific Earthquake Engineering Research Center (PEER) [16], Record \#: NGA0779, ATH: LOMAP/LGP-UP.

is said to be non-stationary if its probabilistic properties vary in time. There are, of course, varying degrees of non-stationarity. We specifically focus on a class of non-stationary processes whose second-order statistics (correlations / power spectra) are time dependent. In the physical interpretation of the SRM, this means that the frequency content is time varying. Practical examples of non-stationary processes are ubiquitous, even when limited just to the fields of civil engineering or applied mechanics. Seismic ground motion time histories, for example, exhibit both amplitude and frequency modulation; as demonstrated in Figure 1 which shows a clear amplitude and frequency modulation. The latter is more readily observed in Figure 1(b) where it can be seen, qualitatively, that the predominant frequencies in the two displayed time windows are significantly different - the top having higher frequency content than the bottom. As another example, Functionally Graded Materials (FGMs) are a class of composites where a gradient exists in stochastic material morphology and properties. FGMs occur both naturally (e.g. bamboo possesses a radially increasing fiber density to resist wind induced bending moments $[17,18]$ ) and are synthetically engineered (Figure 2 shows a two phase aluminum - high density polyethylene composite with varying volume fraction). To capture this spatial variation, the morphology may be characterized by a non-homogeneous

as the theory utilized herein is analogous for both random processes and random fields. 


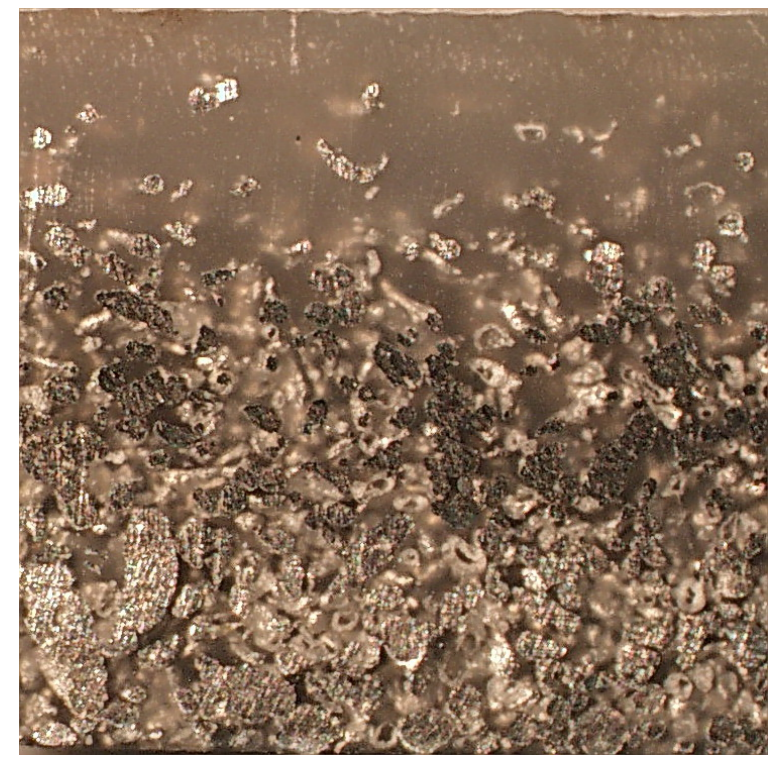

Figure 2: Sample functionally graded material : aluminum particulates in a high density polyethylene matrix (AL-HDPE). Image courtesy of Po-Hua Lee, Columbia University, 2013

stochastic field.

Modeling non-stationary processes represents a particularly challenging problem for which several approaches have been proposed. Limiting these approaches to those possessing a direct physical interpretation (i.e. frequency-based), we see that several different theories for modeling the evolving spectral characteristics exist including, most notably the Instantaneous Power Spectrum [19], the Wigner-Ville Spectrum [20, 21, 22], wavelet transforms [23, 24, 25, 26, 27, 28, 29, 30], and Priestley's theory of evolutionary power [31]. Among these, Priestley's theory is particularly useful because its defining quantity, the evolutionary power spectral density (or evolutionary spectrum - ES) preserves the features of the classical stationary power spectral density as discussed in the following.

Consider a zero mean non-stationary stochastic process $X(t)$ possessing autocorrelation function $(\mathrm{ACF}) R(s, t)$ that admits a representation of the form [31]:

$$
R(s, t)=E[X(s) X(t)]=\int_{-\infty}^{\infty} \phi(s, \omega) \phi^{*}(t, \omega) d \mu(\omega)
$$

where $\phi(t, \omega)$ represents a "family" of functions defined on the real line and $\mu(\omega)$ is a measure 
also defined on the real line. In general, $X(t)$ can be expressed as:

$$
X(t)=\int_{-\infty}^{\infty} \phi(t, \omega) d Z(\omega)
$$

where $Z(\omega)$ is an orthogonal process with:

$$
E\left[|d Z(\omega)|^{2}\right]=d \mu(\omega)
$$

For processes defined on a finite interval (i.e. $0 \leq t \leq T$ ), such a representation always exists with $\phi(t, \omega)$ denoting the eigenfunctions of the covariance kernel; which serves as the basis of the Karhunen-Loève decomposition. The theory of evolutionary power, on the other hand, enforces a family of amplitude modulated complex exponentials such that:

$$
\phi(t, \omega)=A(t, \omega) e^{i \theta(\omega) t}
$$

where $A(t, \omega)$ is the so-called modulating function that can be expressed as:

$$
A(t, \omega)=\int_{-\infty}^{\infty} e^{i \theta t} d H(\omega, \theta)
$$

If $|d H(\omega, \theta)|$ possesses an absolute maximum at $\theta=0$, the process is called oscillatory. Thus, if $\theta(\omega)$ is a single-valued function of $\omega$, the oscillatory process $X(t)$ may be expressed:

$$
X(t)=\int_{-\infty}^{\infty} A(t, \omega) e^{i \omega t} d Z(\omega)
$$

and the ACF takes the form:

$$
R(s, t)=\int_{-\infty}^{\infty} A(s, \omega) A^{*}(t, \omega) e^{i \omega(s-t)} d \mu(\omega) .
$$

Given the oscillatory process defined in Eq. (6), the evolutionary spectrum is defined as:

$$
d S(t, \omega)=|A(t, \omega)|^{2} d \mu(\omega)
$$

The issue addressed in this paper is that the equation defining the autocorrelation function in terms of the evolutionary spectrum (Eq. (7)) is not uniquely invertible. Rather, 
Priestley demonstrates that for any particular oscillatory process $X(t)$ having the form in Eq. (6) "there will, in general, be a number of different families of oscillatory functions in terms of which $X(t)$ has the representation in [Eq. (6)], with each family inducing a different measure $\mu(\omega)$ " [32]. Moreover, Priestley suggests that it is convenient to "standardize" the modulating functions $A(t, \omega)$ such that $A(t=0, \omega)=1 \forall \omega$. This has the effect of incorporating $|A(0, \omega)|$ into the measure $\mu(\omega)$ such that $d \mu(\omega)$ represents the ES at $t=0$ and $|A(t, \omega)|^{2}$ represents the change of the spectrum over time.

Consequently, given a non-stationary autocorrelation function, there is no existing method to determine the ES, in general (analytically or numerically). There exist some techniques to estimate the ES, though these depend on very limiting conditions such as local stationarity or narrow-bandness $[24,28,29,33]$. Identifying such a unique ES has significant practical importance; particularly in the field of stochastic process simulation. Specifically, the spectral representation method (SRM) [1], discussed previously for generation of non-stationary processes, relies on knowledge of the evolutionary spectrum.

To explore the possibility of computing a unique ES from a non-stationary ACF, the inversion of Eq. (7) is posed as a discrete constrained optimization problem. Two different methods are proposed for solving this optimization problem and compared to some "off-theshelf" optimization packages. The solutions obtained from this optimization problem are then used to critically investigate three questions:

1. Is there a solution to the inverse transformation: $R(s, t) \rightarrow S(t, \omega)$ ?

2. If a solution exists, does it give the correct evolutionary spectrum?

3. If a solution exists, is it unique?

In short, as shown later, the results of the analysis suggest that a unique solution is likely to exist although, given that an analytical inversion does not exist, the answer cannot be proven definitively.

\section{Formulation of discrete optimization problem}

In the most general case, shown in Eqn. (7), Priestley [31] has proven that no unique inversion of the autocorrelation function exists to compute the evolutionary spectrum. Here 
the inversion under a certain set of conditions is treated as a discrete optimization problem. We confine ourselves to the case where $A(t, \omega)$ is a real function and $\mu(\omega)=1$. Under these conditions, the evolutionary spectrum can be expressed as:

$$
S(t, \omega)=A^{2}(t, \omega)
$$

and the integral in Eqn. (7) reduces to:

$$
R(s, t)=\int_{-\infty}^{+\infty} A(s, \omega) A(t, \omega) e^{i \omega(s-t)} \mathrm{d} \omega
$$

Note, also, that because the modulating function $A(t, \omega)$ and the evolutionary spectrum $S(t, \omega)$ completely define each other in this case, the two will be used interchangeably throughout.

Considering a simple substitution of variables such that $\tau=s-t$ defines a lag, the ACF is discretized as:

$$
R_{j k}=R\left(t_{j}, \tau_{k}\right)=R(j \Delta t, k \Delta \tau)
$$

and the modulating function is discretized as:

$$
A_{j k}=A\left(t_{j}, \omega_{k}\right)=A(j \Delta t, k \Delta \omega)
$$

Often, $\Delta t=\Delta \tau$ although this is not a necessary condition. The discrete optimization problem considered in this work is given by:

$$
\begin{array}{ll}
\underset{R^{c}}{\operatorname{minimize}} & \varepsilon=\frac{\left\|R_{j k}^{t}-R_{j k}^{c}\right\|}{\left\|R_{j k}^{t}\right\|} \\
\text { subject to: } & A_{j k} \geq 0, \forall j, k
\end{array}
$$

where $R^{t}$ is the prescribed target autocorrelation function, $R^{c}$ is the estimated autocorrelation function and the following norm is adopted:

$$
\left\|X_{j k}\right\|=\sqrt{\sum_{j=1}^{N_{t}} \sum_{k=1}^{N_{\tau}} X_{j k}^{2}}
$$


possessing

$$
\begin{aligned}
N_{t} & =\frac{t_{\text {max }}}{\Delta t} \\
N_{\tau} & =\frac{\tau_{\max }-\tau_{\min }}{\Delta \tau}
\end{aligned}
$$

Stated less formally, we would like to find some $A(t, \omega)$ that, when integrated as in Eqn. (10), approximates as closely as possible the target $\mathrm{ACF}, R^{t}(t, \tau)$. By discretizing the modulating function and autocorrelation as in Eqns. (11) and (12), the integral in Eqn. (10) can be evaluated numerically to find $R_{j k}^{c}$. An additional challenge of this optimization problem, beyond the question of existence and uniqueness, is the very high dimension. Notice that the optimization is occurring over a dual-indexed discretized function possessing $N_{t} \times$ $N_{\omega}$ points, where $N_{\omega}$ is the number of discrete points in the frequency domain. It is not uncommon for $N_{t}$ and $N_{\omega}$ to take values of, say 1,024, in which case the optimization space has dimension 1,048,576. Given this extremely high dimensionality, many conventional optimization algorithms will either fail to converge or will be computationally intractable. For this reason, two new approaches are presented in the following sections that have been specifically designed to solve this high dimensional optimization problem and ultimately attempt to answer the existence and uniqueness questions posed in the previous section.

\section{Perturbation method (PM)}

\subsection{Description of algorithm}

The first method, referred to as the Perturbation Method (PM), was first proposed by Shields [34] and has also been used by Shields to generate artificial non-stationary ground motion time histories that are compatible with a prescribed response spetrum [35]. In short, it involves the iterative random perturbation of the evolutionary spectrum and either acceptance or rejection of the perturbations depending on improvement or worsening of the error $\varepsilon$ in Eq. (13). Next, the detailed procedure for the PM will be presented.

The PM proceeds as follows and is illustrated in Figure 3.

1. Prescribe the target autocorrelation function, $R^{t}(t, \tau)$. 


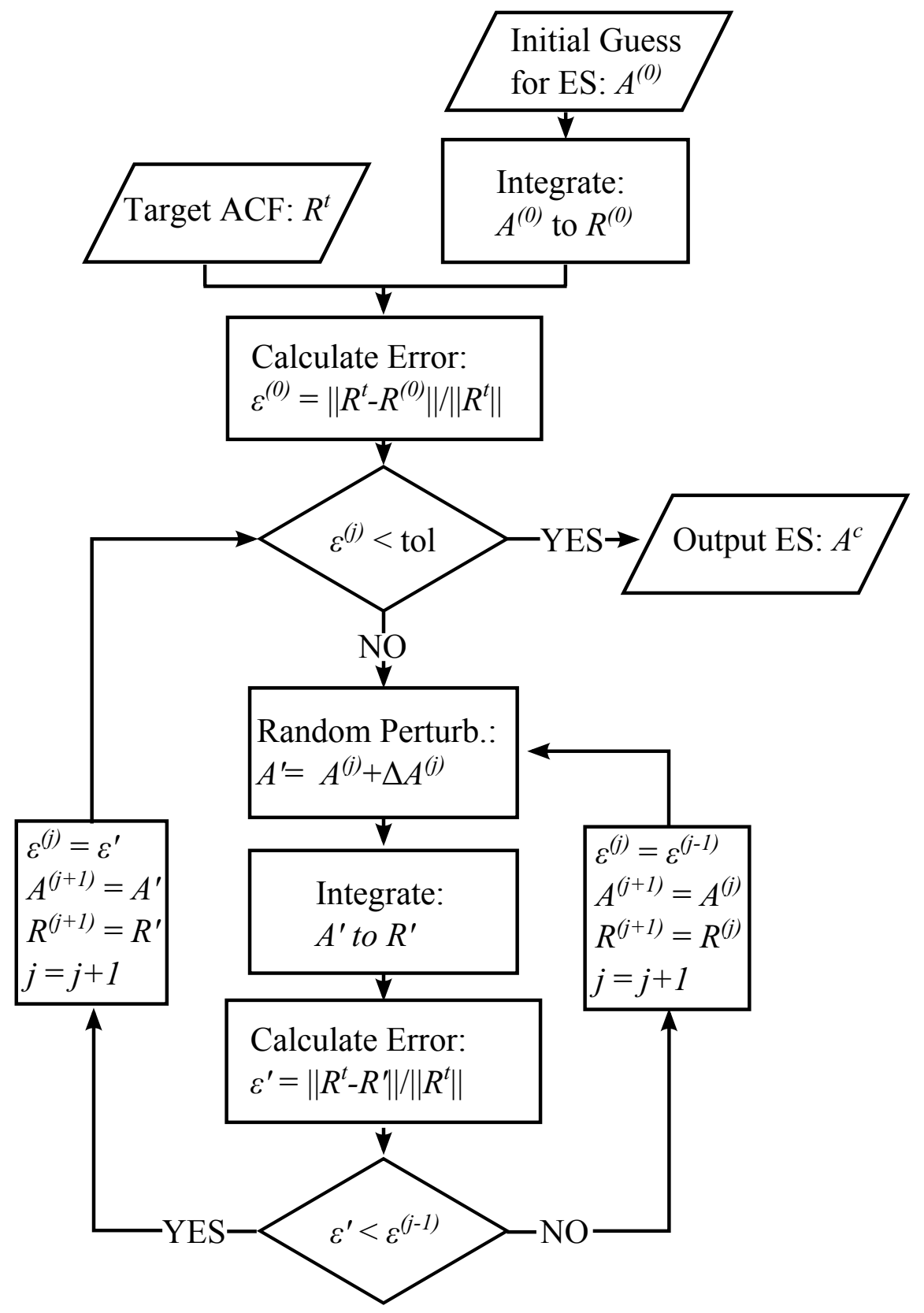

Figure 3: Perturbation Method (PM) algorithm 
2. Initialize the evolutionary spectrum, $A^{0}(t, \omega)$, with arbitrarily selected positive values for all $t, \omega$ (note that selection of a good initial ES is discussed in Section 5).

3. Integrate the ES to determine the ACF, $R^{c}(t, \tau)$, using Eq. (10).

4. Compute the error $\varepsilon$ between the target $\mathrm{ACF}, R^{t}(t, \tau)$, and the computed $\mathrm{ACF}, R^{c}(t, \tau)$, using Eq. (13).

5. Perturb the ACF as follows:

$$
A^{(j)}(t, \omega)=A^{(j-1)}(t, \omega)+\Delta A^{(j)}(t, \omega)
$$

where

$$
\Delta A^{(j)}(t, \omega)=C^{(j)} \exp \left(-\frac{\left(t-t^{\prime}\right)^{2}}{\alpha_{t}^{(j)}}\right) \cdot \exp \left(-\frac{\left(\omega-\omega^{\prime}\right)^{2}}{\alpha_{\omega}^{(j)}}\right),
$$

$t^{\prime}, \omega^{\prime}$ are random time and frequency values uniformly distributed in the intervals $[0, T]$ and $\left[0, \omega_{u}\right]$ respectively, $C^{(j)}$ is a (iteration varying) magnitude scale parameter, and $\left(\alpha_{t}^{(j)}, \alpha_{\omega}^{(j)}\right)$ are "width" control parameters (also iteration dependent). Discussion of parameter selection follows in Section 3.2.

6. Integrate the ES to determine the $\mathrm{ACF}, R^{c}(t, \tau)$, using Eq. (10).

7. Compute the error $\varepsilon$ between the target $\mathrm{ACF}, R^{t}(t, \tau)$, and the computed $\mathrm{ACF}, R^{c}(t, \tau)$ at iteration $(j)$, using Eq. (13).

8. If the perturbation $\Delta A^{(j)}(t, \omega)$ reduces the error, i.e. $\varepsilon^{(j)}<\varepsilon^{(j-1)}$, then the perturbation is accepted. Otherwise, the perturbation is rejected and $A^{(j)}(t, \omega)=A^{(j-1)}(t, \omega)$.

9. Iterate back to step 5 and repeat until $\varepsilon<\varepsilon_{t h}$ where $\varepsilon_{t h}$ is a user specified error threshold.

For more discussion of this method see [34]. The method is conceptually similar to the simulated annealing (SA) method [36, 37, 38, 39] with some basic differences. The error is referred to as the energy in the SA method. There are two primary differences between the PM and SA. The first is that the PM relies on a functional perturbation rather than a point wise perturbation. The second is that that PM does not rely on the SA concept of "temperature." The importance of these differences will be discussed in the following section. 


\subsection{Discussion}

The perturbation method detailed in the previous section applies repeated random perturbations, the details of which are essential to its convergence and provide insight into the existence and uniqueness of the evolutionary spectrum. These details are discussed here.

The perturbations of the form in Eq. (17) have several important features. First, as previously discussed, they follow a functional form as opposed to point-wise perturbations applied using simulated annealing. This form is selected for two reasons: to preserve a degree of smoothness in the evolutionary spectrum, and to enable the simultaneous perturbation of numerous points in the space. The latter is important given the very high dimension of the optimization problem. The appropriate selection of the perturbation function parameters is essential as well. The magnitude scaling parameter, $C^{(j)}$, is chose to be a fraction of $A\left(t^{\prime}, \omega^{\prime}\right)$ with random sign (power can be added or removed). Its value can decrease in magnitude as the iterations progress to allow "fine-tuning" of the evolutionary spectrum.

Similarly, the width parameters $\left(\alpha_{t}^{(j)}, \alpha_{\omega}^{(j)}\right)$ can be reduced as the iterations progress to produce increasingly narrow perturbations; thus allowing more localized adjustments in the later iterations.

Convergence to the evolutionary spectrum using the PM does not prove uniqueness, though it is a strong indicator of such. Because the convergence path is stochastic, repeated convergence to the same ES implies uniqueness (though not a rigorous proof, of course). Moreover, the lack of a need for a "temperature" parameter, as in simulated annealing, suggests that the error function being minimized, Eq. (13), is convex. In SA, the temperature parameter represents a probability of acceptance/rejection given that the error is reduced/increased during a given iteration and it usually changes as the iterations progress to allow fewer "false acceptances/rejections" at later iterations. Its intention is to allow some small number of rejections even when the error is reduced and some small number of acceptances even when the error increases in order to allow the solution to climb out of local minima. The lack of a need for temperature (or perhaps more appropriately a constant unit temperature) in the PM suggests that no evolutionary spectrum exists that will produce a 


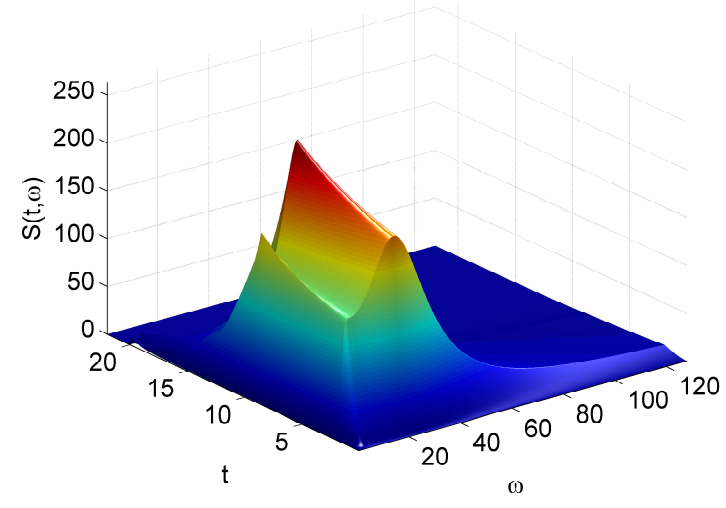

(a) ES

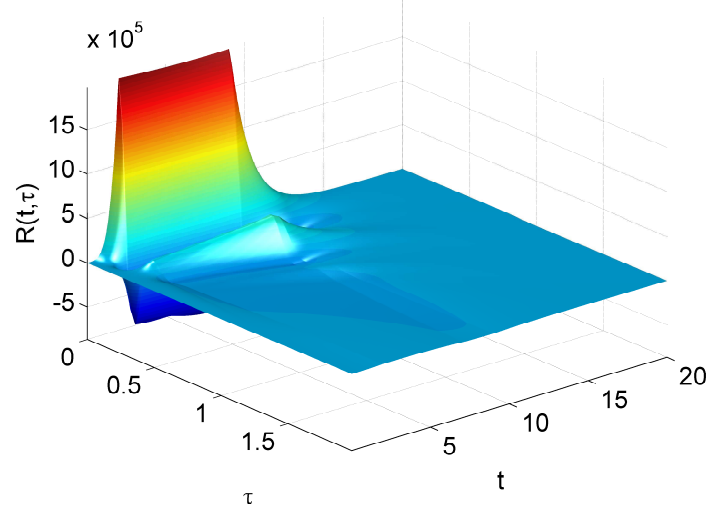

(b) $\mathrm{ACF}$

Figure 4: Clough-Penzien (CP) spectrum with amplitude and frequency modulation and corresponding non-stationary autocorrelation function

local minimum in the error function that is not also a global minimum - essentially precluding the ES from converging to solutions that take different shapes.

\subsection{PM results}

In this example and all subsequent examples, we will examine convergence by estimating the ES from the ACF for a known example ES. Here, we consider an ES model used for the simulation of non-stationary seismic ground motion [40] - specifically the Kanai-Tajimi acceleration spectrum with Clough-Penzien correction [41] possessing both frequency and amplitude modulation [42]. This spectrum is shown in Figure 4(a), along with the corresponding ACF as computed by Eq. (10) in Figure 4(b), and is defined as:

$$
S(\omega, t)=M^{2}(t) \underbrace{S_{0}(t)\left[\frac{1+4 \zeta_{g}^{2}\left(\frac{\omega}{\omega_{g}}\right)^{2}}{\left[1-\left(\frac{\omega}{\omega_{g}}\right)^{2}\right]^{2}+4 \zeta_{g}^{2}\left(\frac{\omega}{\omega_{g}}\right)^{2}}\right]}_{\text {Non-stationary Kanai-Tajimi spectrum }} \times \underbrace{\left[\frac{\left(\frac{\omega}{\omega_{f}}\right)^{4}}{\left[1-\left(\frac{\omega}{\omega_{f}}\right)^{2}\right]^{2}+4 \zeta_{f}^{2}\left(\frac{\omega}{\omega_{f}}\right)^{2}}\right]}_{\text {Clough-Penzien correction }}
$$

where the amplitude modulation is given through the Jennings, Housner, and Tsai modulating function [43]:

$$
M(t)= \begin{cases}\left(\frac{t}{t_{1}}\right)^{2} & 0 \leq t \leq t_{1} \\ 1 & t_{1} \leq t \leq t_{2} \\ \exp \left[-c\left(t-t_{2}\right)\right] & t_{2} \leq t\end{cases}
$$




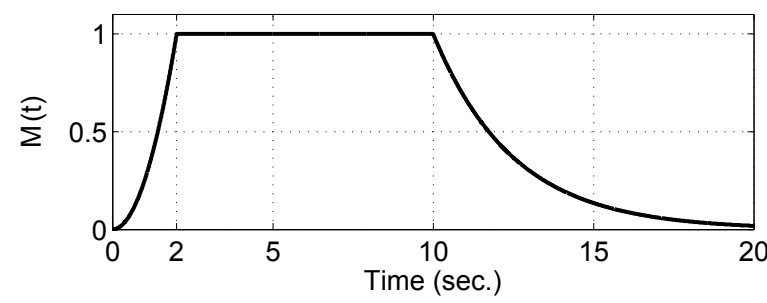

Figure 5: Jennings, Housner, and Tsai modulating function [43]

which is shown in Figure 5. The parameter $S_{0}(t)$ in Eqn. (18) is used to describe the intensity of the acceleration in the underlying Kanai-Tajimi spectrum [44, 45]. It is defined as:

$$
S_{0}(t)=\frac{\sigma^{2}}{\pi \omega_{g}\left(\frac{1}{2 \zeta_{g}}+2 \zeta_{g}\right)}
$$

where $\sigma$ is the standard deviation, and $\omega_{g}$ and $\zeta_{g}$ in Eqns. (18) and (20) are the characteristic frequency and damping, respectively, of the ground. Ellingwood and Batts [46] have suggested values for rock, deep cohesionless soils, clays, and sands. Finally, $\omega_{f}$ and $\zeta_{f}$ in Eqn. (18) are the filtering parameters of the Clough-Penzien correction [41], which are typically taken to be:

$$
\begin{aligned}
\omega_{f} & =0.1 \omega_{g} \\
\zeta_{f} & =\zeta_{g}
\end{aligned}
$$

The parameter definitions used in this numerical example are as follows:

$$
\begin{aligned}
\omega_{g} & =30\left[\frac{\mathrm{rad}}{\mathrm{sec}}\right]-1.25\left[\frac{\mathrm{rad}}{\mathrm{sec}^{2}}\right] t \\
\zeta_{g} & =0.5\left[\frac{\mathrm{rad}}{\mathrm{sec}}\right]+0.005\left[\frac{\mathrm{rad}}{\mathrm{sec}^{2}}\right] t \\
\sigma & =110\left[\frac{\mathrm{cm}}{\mathrm{sec}^{3 / 2}}\right] \\
t_{1} & =2 \mathrm{sec} \\
t_{2} & =10 \mathrm{sec} \\
c & =0.4
\end{aligned}
$$




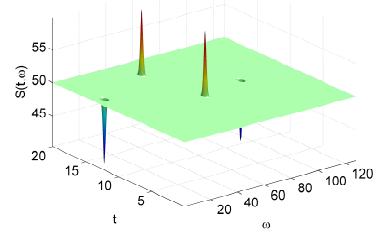

(a) 10 iterations

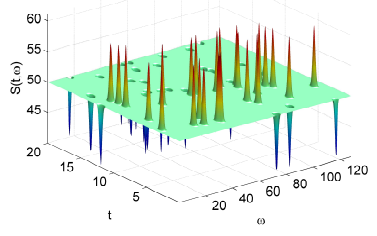

(b) 100 iterations

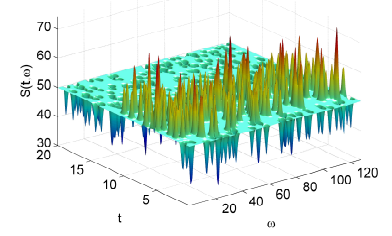

(c) 1000 iterations

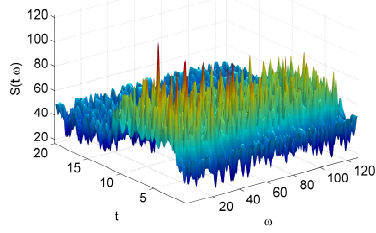

(d) 10000 iterations

Figure 6: Progression of the PM over the first 10,000 iterations

The initial guess for the evolutionary spectrum was a uniform spectrum with $A(t, \omega)=$ $50 \forall(t, \omega)$. Time and frequency were discretized as:

$$
\begin{aligned}
t & \in(0,20) \mathrm{sec} \\
\tau & \in(0,2) \mathrm{sec} \\
\Delta t & =0.025 \mathrm{sec} \\
\Delta \tau & =0.025 \mathrm{sec} \\
\omega & \in(0,128) \mathrm{rad} / \mathrm{sec} \\
\Delta \omega & =0.5 \mathrm{rad} / \mathrm{sec}
\end{aligned}
$$

The PM was applied for 1,000,000 iterations with Figure 6 showing a typical progression of perturbations resulting in the ES shown in Figure 7(a). A "slice" (at constant $t$ ) comparing the estimated ES with the target ES is shown in Figure 7(b). The associated ACF, computed using Eq. (10) is shown in Figure 7(c). Lastly, the progression of error is shown in Figure 7(d) with a final error of $1.57 \%$ when measured according to Eqn. (13). Measured in the ES, the error is $6.95 \%$ which is a byproduct of the "bumpy" solution obtained through perturbations of the form in Eq. (17). Visually, both the ACF and ES are remarkably close in their general shape when compared to their respective targets (Figure 4). The ACF is noticeably smoother than the ES, and matches the target quite nicely - as evident quantitatively through the error measure, as well. This suggests that the ACF is largely dependent on the general shape of the ES and fairly insensitive to the fluctuations around that shape.

Returning to the primary intentions listed at the beginning of this section, these results show clear convergence to a specific, unique evolutionary spectrum with the correct general 


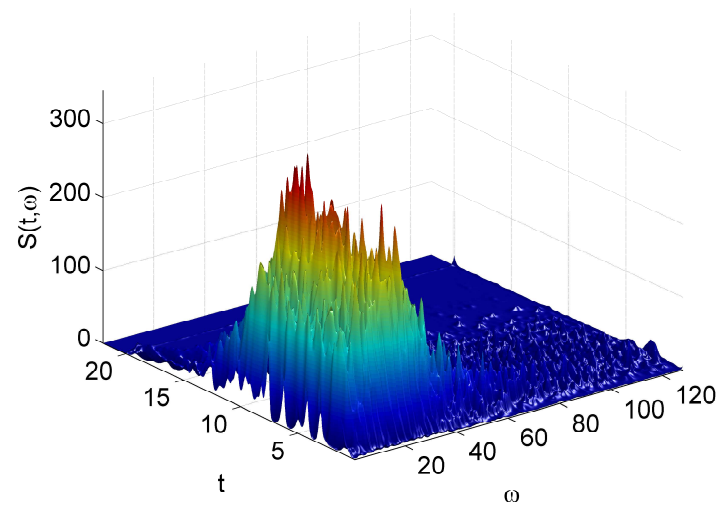

(a) PM estimated ES

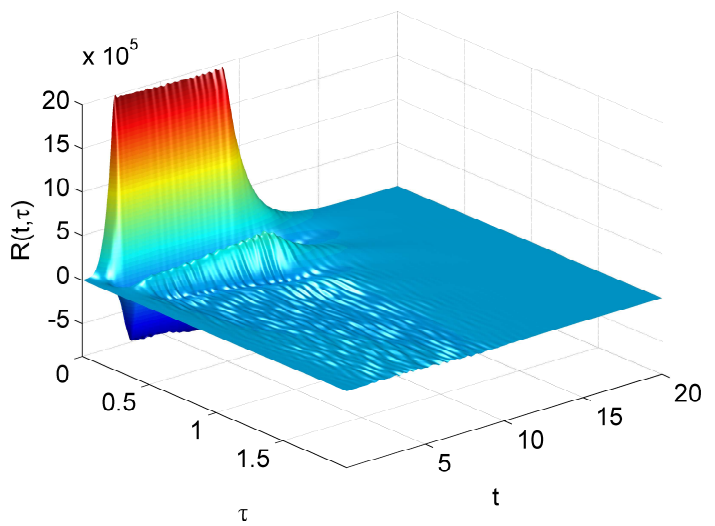

(c) PM estimated ACF

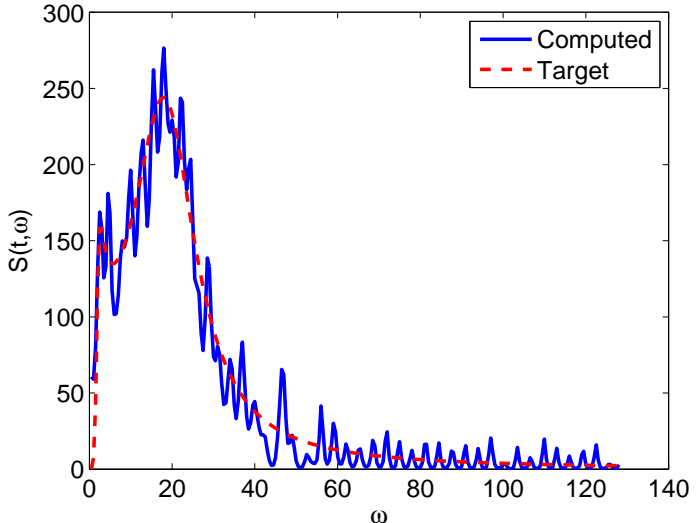

(b) PM estimated ES "Slice"

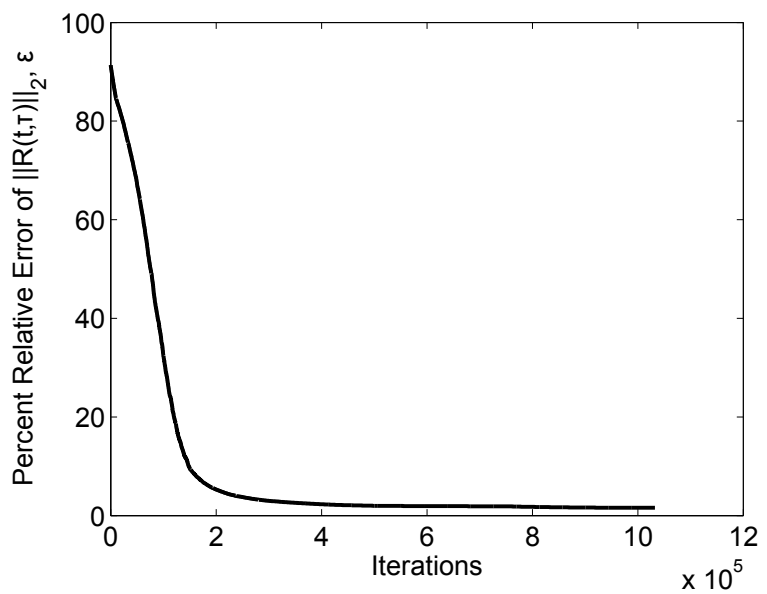

(d) PM errors vs. iteration

Figure 7: PM convergence results for Clough-Penzien (CP) spectrum with amplitude and frequency modulation. After 1,000,000 iterations of PM, final error: $6.95 \%$ in ES and 1.57\% in ACF 


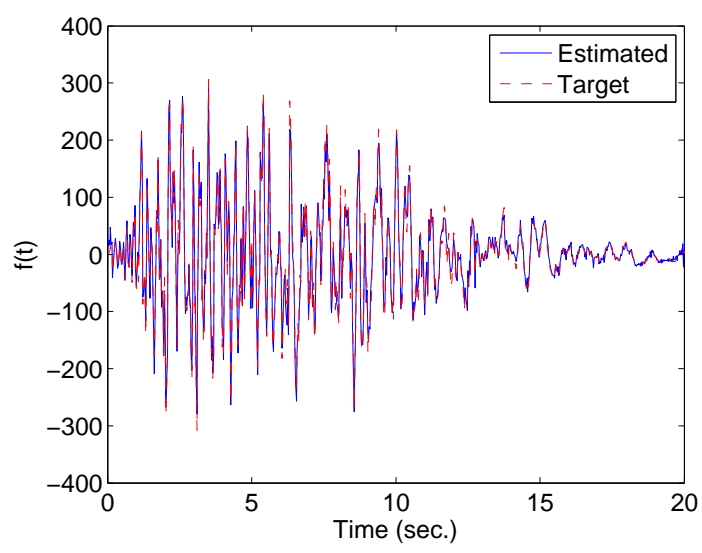

(a) Simulated sample realization comparison
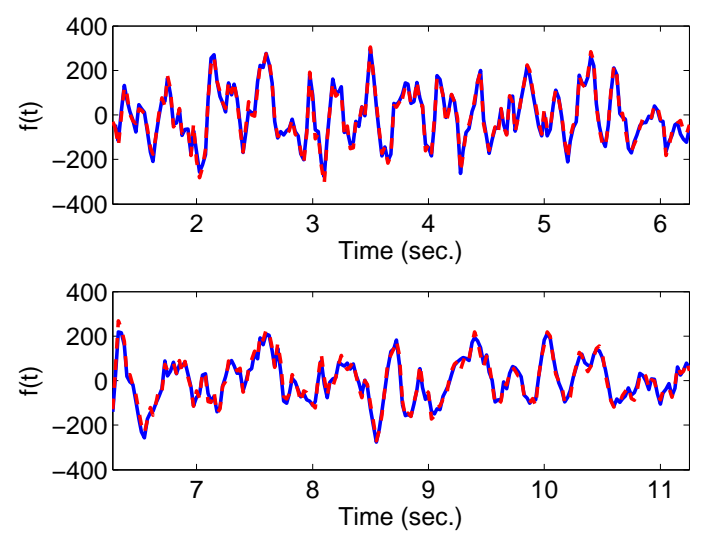

(b) Zoom in on sample realizations

Figure 8: Comparison of sample realizations generated using SRM with target ES (red dash) and estimated ES (solid blue)

shape. Specifically, through convergence to a clearly defined shape, and not an arbitrary shape, the results point to the likelihood that a solution exists. The repeated convergence to similar solutions has been observed (including similar convergence shown in [34]) and points to the uniqueness of this solution. Note that the convergence path is stochastic, and there is no bias in the algorithm. In other words, there are no a priori assumptions (as to the shape or behavior of the ES) nor any information other than the ACF used. Thus, if the same minimum is found from repeated application of the method, and is the only minimum found, it is very likely that this is the only minimum that exists. Although this has not been performed for a very large number of times due to computational cost, the method has been employed numerous times with consistent results.

Figure 8 shows sample realizations generated using both the target and estimated evolutionary spectra using the spectral representation method. The two samples were generated using the same random phase angles, for direct comparison. Figure $8(\mathrm{a})$ shows the entire 20 second duration, and Figure 8(b) shows two 5 second window zoom-ins. It is clear that the sample generated from the estimated spectrum matches the target quite well. The noise in the estimated spectrum (7(a)) appears to have minimal effect on the generated samples. This indicates that identifying the general shape of the spectrum may be sufficient for the simulation of many stochastic processes. 


\section{4. "Multi-Grid" Method (MGM)}

\subsection{Description of algorithm}

In the previous section it was shown, though not proven, that a unique solution to the inversion of Eqn. (10) likely exists. The PM described therein was certainly not efficient enough for most practical applications; the computation to sufficient accuracy takes several days on a standard PC. To that end, the remainder of this paper aims to make this computation more efficient. This section develops and examines a proposed "Multi-Grid" method, and carries out numerical examples of such. The proposed method is then compared against existing "off-the-shelf" optimization techniques.

In order to increase the efficiency of the convergence, the proposed "Multi-Grid" Method aims to reduce the parameter space of the problem. The example studied for the PM was on a discrete space of $800 \times 256$ 'parameters' to be estimated; producing a parameter space with more than 200,000 degrees of freedom. With uniform random sampling for the perturbations, a minimum of 400,000 iterations, on average, is needed just to ensure each location is perturbed just once. Often the domain will be discretized even further. If the original domain is, for example, $1024 \times 1024$, then the parameter space possesses over 1,000,000 degrees of freedom. However, by using interpolation and assuming some level of continuity, this space can be significantly reduced. Furthermore, inspired by the multi-grid solvers used in linear systems [47, 48], it will be demonstrated that it is possible to use the information on a coarse-scale grid to improve convergence on a fine-scale grid.

The MGM algorithm is shown in Figure 9. The algorithm is similar to the PM described previously, with the addition of a mesh refinement step. The mesh begins coarse (e.g., for the examples in Section 4.2 a $20 \times 10$ initial mesh is used) and gradually increases in density to the finest scale $(800 \times 256)$ over the course of the iterations. When the mesh is transfered from coarser to finer, bi-cubic spline interpolation is used. This assumes a certain degree of continuity and smoothness, as the bi-cubic splines preserve $C^{2}$-continuity. However, splines do not preserve non-negativeness, which is a constraint on the evolutionary spectrum. To correct this, local linear interpolation is used if any negative values are produced by the spline interpolation. Furthermore, although the random perturbations are carried out on 


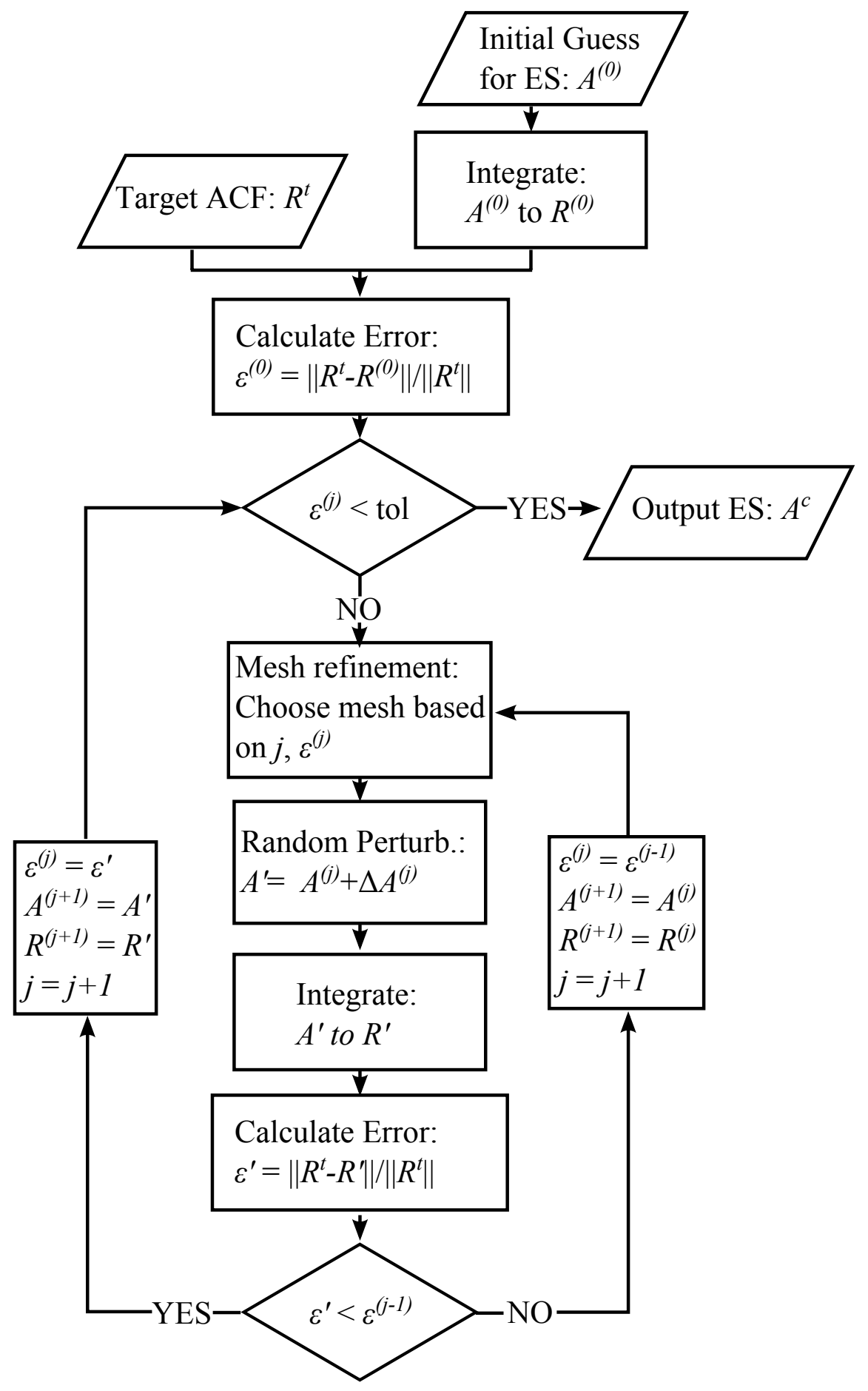

Figure 9: Multi-Grid Method (MGM) algorithm 
the coarse mesh, the errors are always calculated on the fine scale. Otherwise, the norm defined in Eqn. (14) would not be consistent between different meshes.

\subsection{MGM results}

In this section, two numerical examples are carried out using the MGM. First, the KT-CP example used earlier in the PM for seismic ground motion modeling is revisited. Then, an arbitrary and non-physical spectrum is considered to demonstrate the robustness of the algorithm. In the first case, convergence is compared with various "off-the-shelf" optimization techniques.

\subsubsection{Seismic ground motion example}

In the first numerical example, the KT-CP spectrum from Section 3.3 is considered. The parameters and initial guess are identical to that example, though in this case $\sigma=100$. Figure 10 shows the progression of the MGM over the first 1,000 iterations. In contrast to the PM shown in Figure 6, improvements are recognized much more quickly. Because the perturbations are on the coarse scale, each perturbation affects a wider set of points in the (fine scale) domain. Early in the iterations, this is particularly useful in identifying the general shape faster. As such, power begins to accumulate in the correct areas much sooner in the MGM than the PM, and by 1,000 iterations the general shape is starting to form; for the PM this was taking 10,000 or more iterations. The resulting evolutionary spectrum (ES) is shown in Figure 11(a), and a sample slice is shown in Figure 11(b). The associated autocorrelation function (ACF) is shown in Figure 11(c). The convergence behavior is compared to the PM in Figure 11(d). After four hundred thousand iterations, the error measured in the $\mathrm{ACF}$ is $0.6 \%$, and $7.5 \%$ in the ES. To reach an error level equivalent to the one million iterations of the PM, roughly one hundred thousand iterations of the MGM are needed - a full order of magnitude reduction in effort.

In addition to efficiency improvements, the MGM better preserves the continuity and smoothness of the ES than the PM. Qualitatively, it can be seen that the result in Figure 11(a) is quite smooth, and looks closer to Figure 4(a) than the PM result (Figure 7(a)). Comparing the slices of Figures 7(b) and 11(b), the noise from the PM is almost completely 


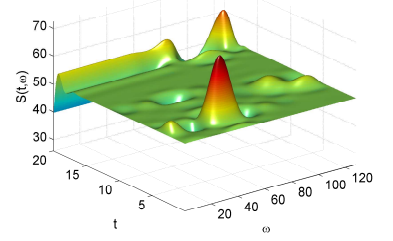

(a) 10 iterations

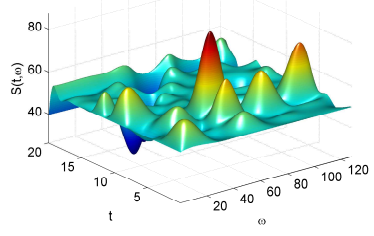

(b) 100 iterations

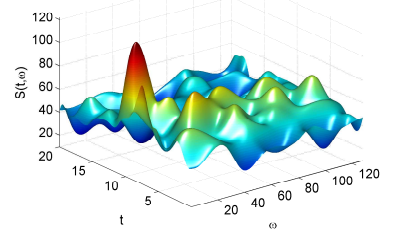

(c) 500 iterations

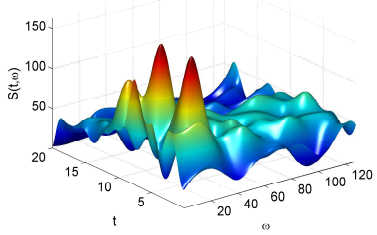

(d) 1000 iterations

Figure 10: Progression of the MGM over the first 1,000 iterations

eliminated using the MGM. This is especially true in the regions of high power (and thus higher importance).

The majority of the error in the ES computed using the MGM is concentrated on the boundaries of the domain. No "boundary conditions" were imposed so as to avoid any bias. But, the target KT-CP evolutionary spectrum possesses $S(t, 0)=0 \forall t$ (i.e. no power at zero frequency) and, for simulation purposes using the SRM, it is assumed that $S\left(t, \omega>\omega_{u}\right)=0$ where $\omega_{u}$ is an upper cutoff frequency. Furthermore, depending on the physical process, $S(t=0, \omega)$ and $S(t=T, \omega)$ can often be assumed to be zero. Thus, either with constraints or post-processing, the ES-based error in the MGM could be improved considerably.

Simulated sample functions generated using the SRM with the MGM-estimated and target spectra are shown in Figure 12. Once again, the samples were generated using the same random phase angles for direct comparison. Figure 12(a) shows the samples for the entire duration, and Figure 12(b) shows two five second windows. There is very good agreement between the two samples, although some additional high-frequency content is present in the estimated sample - a direct result of the boundary effects discussed previously that could be removed through post-processing or imposing constraints on the objective function.

Finally, convergence of the MGM is compared with some established "off-the-shelf" optimization techniques in Figure 13. Specifically, the optimization problem is solved using the Matlab simulated annealing, genetic algorithm, and pattern search utilities. The MGM is clearly superior to the off-the-shelf methods given the enormous parameter-space. These methods are all severely limited in the number of parameters they can optimize, and are not designed for the scale of this problem. Simulated Annealing which has been used for 


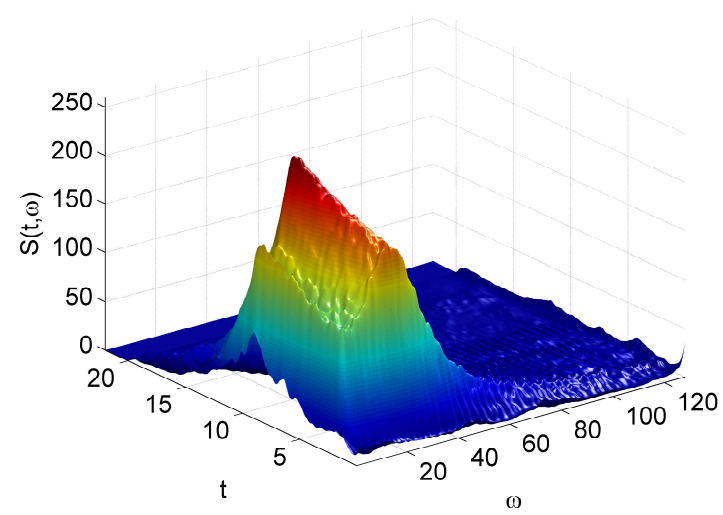

(a) MGM-computed ES

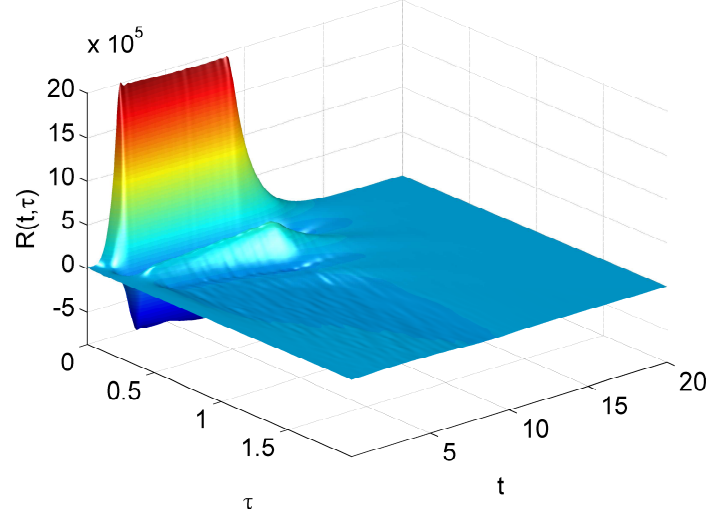

(c) MGM-computed ACF

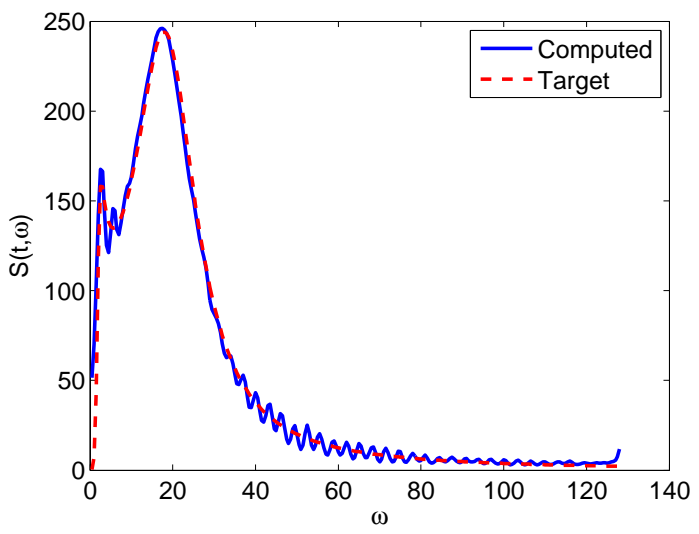

(b) MGM-computed ES "Slice"

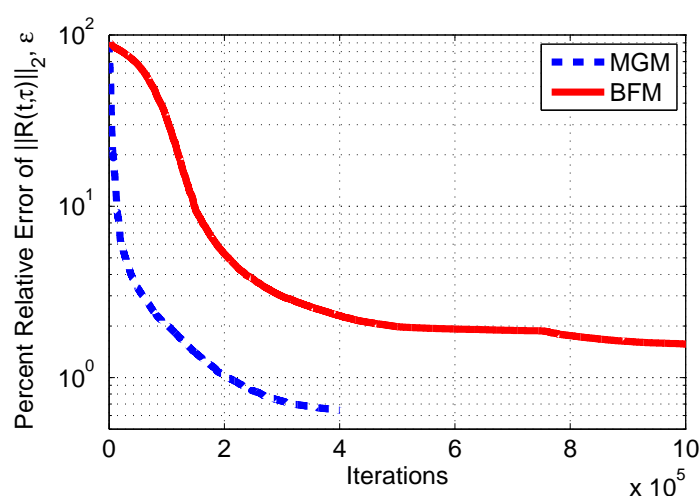

(d) MGM errors vs. iteration

Figure 11: Convergence results for Clough-Penzien (CP) spectrum with amplitude and frequency modulation. After 400,000 iterations of MGM, final error: $7.53 \%$ in ES and $0.64 \%$ in ACF 


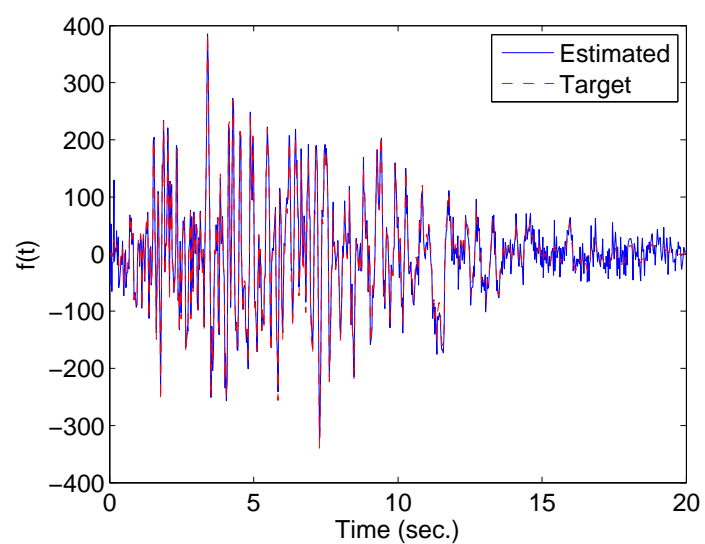

(a) Simulated sample realization comparison
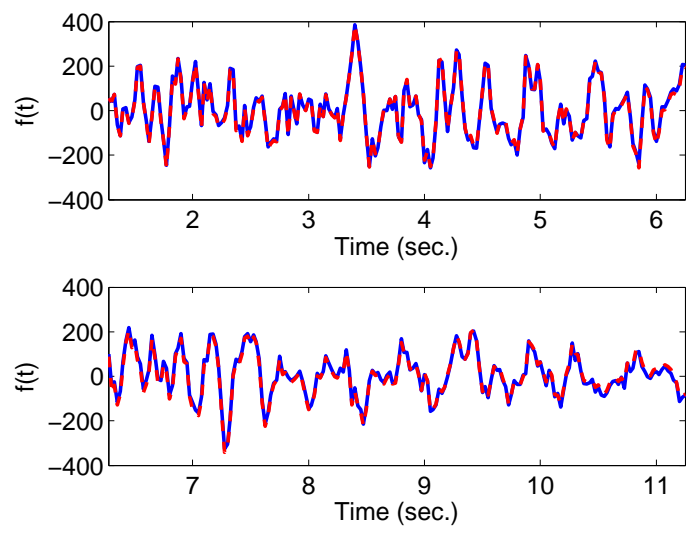

(b) Zoom in on sample realizations

Figure 12: Comparison of sample realizations generated using SRM with target ES (red dash) and MGMestimated ES (solid blue)

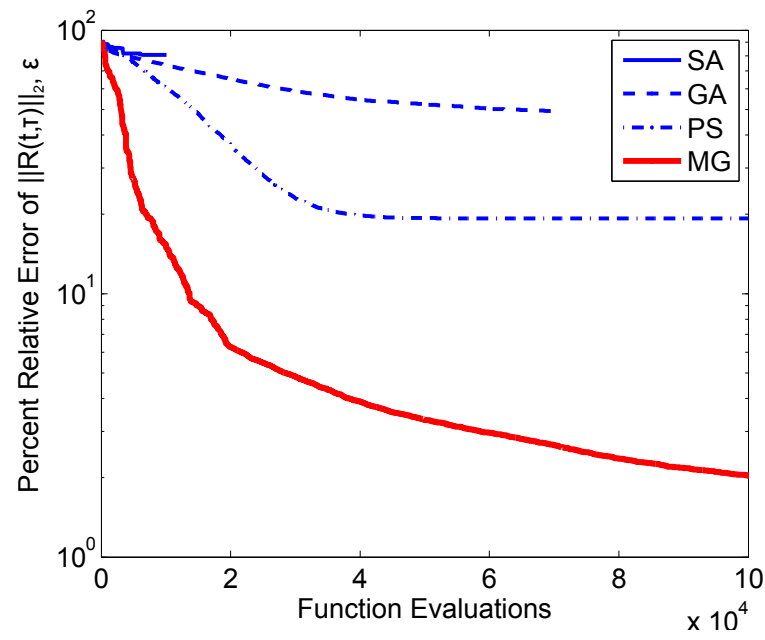

Figure 13: Comparison of the MGM with off-the-shelf optimization techniques: (1) Simulated Annealing (SA), (2) Genetic Algorithm (GA), and (3) Pattern Search (PS) from the MATLAB [49] Global Optimization Toolbox 


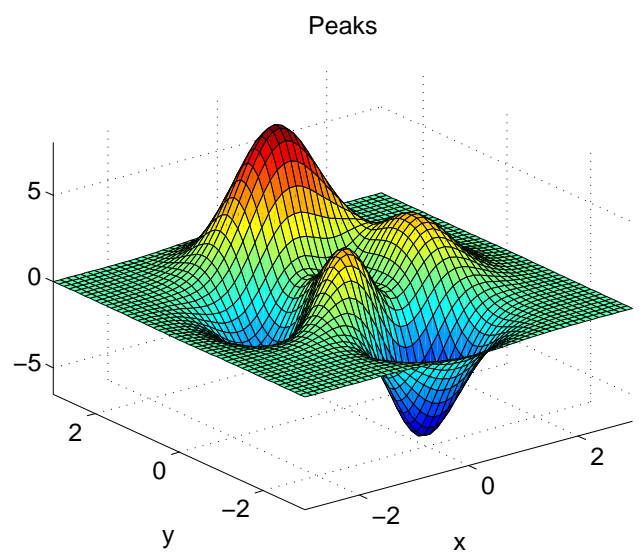

Figure 14: MATLAB peaks function

problems with very large parameter space (e.g. Yeong and Torquato [50] use SA to generate artificial material morphologies) very quickly runs out of memory. The primary difference between the problem posed here and those of the morphology simulations using SA in the literature is that the parameter values in the morphology example are discrete and binary in nature (simulations are for two-phase media) whereas those in the problem posed here are continuous on the space of positive real values possessing smooth functional continuity. The Genetic Algorithm and Pattern Search methods both cease to converge beyond a high error level. In order to make use of these tools, the parameter space would have to be drastically reduced, and in doing so, fine-scale details would be lost.

\subsubsection{MATLAB peaks example}

The second example considers an arbitrary and non-physical evolutionary spectrum defined in order to demonstrate the robustness of the proposed method, as well as the method's ability to capture an arbitrary shape and number of peaks in the ES. The prescribed target evolutionary spectrum is the absolute value of the peaks function in MATLAB [49], which is plotted in Figure 14 and expressed as:

$$
z=3(1-x)^{2} e^{-\left(x^{2}+(y+1)^{2}\right)}-10\left(\frac{x}{5}-x^{3}-y^{5}\right) e^{-x^{2}-y^{2}}-\frac{1}{3} e^{-\left((x+1)^{2}+y^{2}\right)}
$$

where $x$ and $y$ are each defined in $(-3,3)$. The domain has been scaled and translated to match that used in the earlier examples (with the same discretization) yielding the target ES 
and associated ACF shown in Figures 15(a) and 15(c), respectively. After 200,000 iterations, the ES and ACF computed using the MGM are shown in Figures 15(b) and 15(d), exhibiting excellent agreement with their respective targets. The final errors after 200,000 iterations are $0.68 \%$ in the $\mathrm{ACF}$ and $4.58 \%$ in the ES. Once again, the majority of the error in the ES exists at the boundaries. Two time slices of the ES are shown in Figures 15(e) and 15(f) for $t=9.4 \mathrm{sec}$ and $t=13.15 \mathrm{sec}$ respectively. The MGM captures the general shape of the peaks very nicely. It also captures regions of high power very accurately. The primary sources of error in the ES estimation are clearly the boundaries and locations where the absolute value creates weak discontinuities (in the vicinity of $\omega=40 \mathrm{rad} / \mathrm{sec}$ ). Aside from these two problem areas, the representation of the ES by the MGM is virtually perfect. In fact, looking at the convergence behavior in Figure 16, this example converges more rapidly than the previous. It reached errors in the ACF below 1\% before 100,000 iterations. Again, sample realizations are generated using the target ES (Figure 15(a)) and estimated ES (Figure 15(b)) and compared in Figure 17. Figure 17(a) shows the entire period, and Figure 17(b) shows two five second windows. Once again, the MGM estimated ES produces a sample realization that agrees very nicely with the target sample.

\subsection{Computational efficiency}

In the previous section, the MGM was shown to be at least an order of magnitude more efficient than the PM in terms of iterations. However, it is important to consider the full computational cost, comparing the CPU time for both methods. Table 1 shows the average CPU time per iteration for both methods, on a $1024 \times 1024$ grid (note: this is finer than the results presented above). The MGM is only slightly slower per iteration. However, when accounting for the fact that the PM takes roughly 1,000,000 iterations to converge and the MGM 130,000 to converge to the same error level (1.57\%), it is clear that the MGM is still much more efficient than the PM. In both cases, the advantage of parallelization is clear - improvements of a full order of magnitude are possible by parallelizing on a desktop PC. Unfortunately, the algorithms are not fully parallelizable due to the interdependence of iterations. However, computation of the integral in Eqn. (10) can be parallelized because 


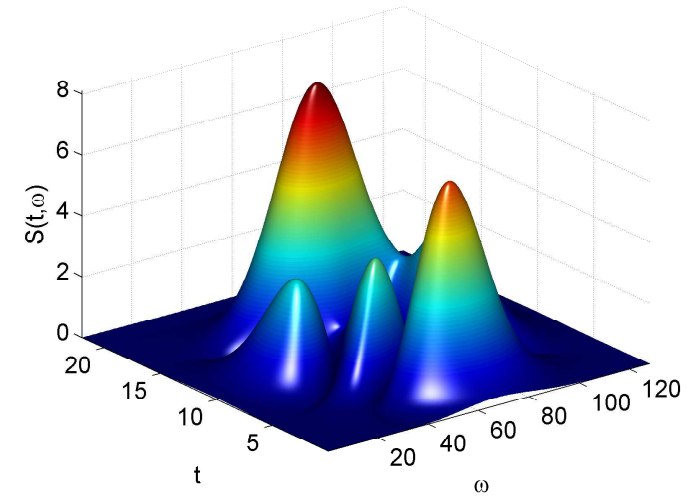

(a) Target ES

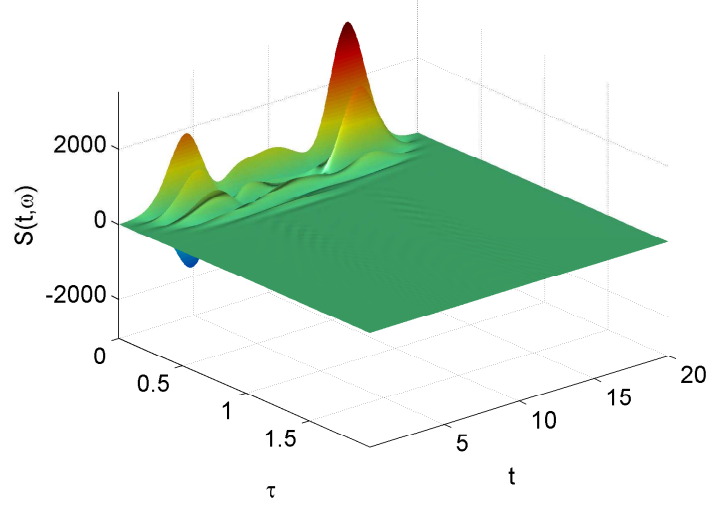

(c) Target ACF

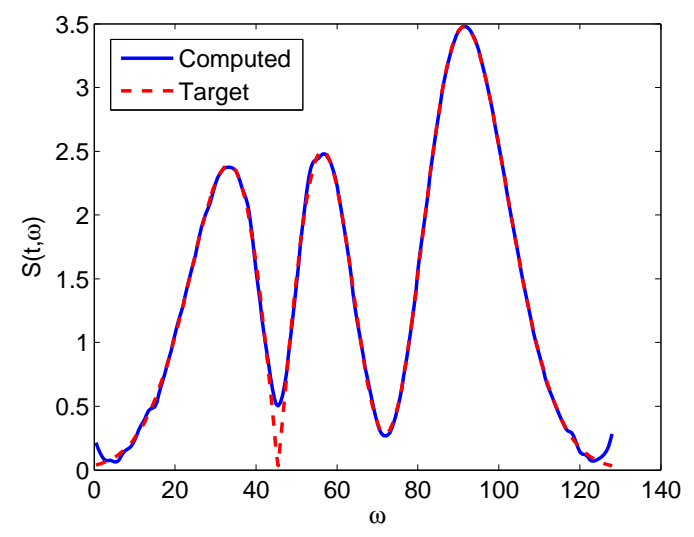

(e) Sample ES slice

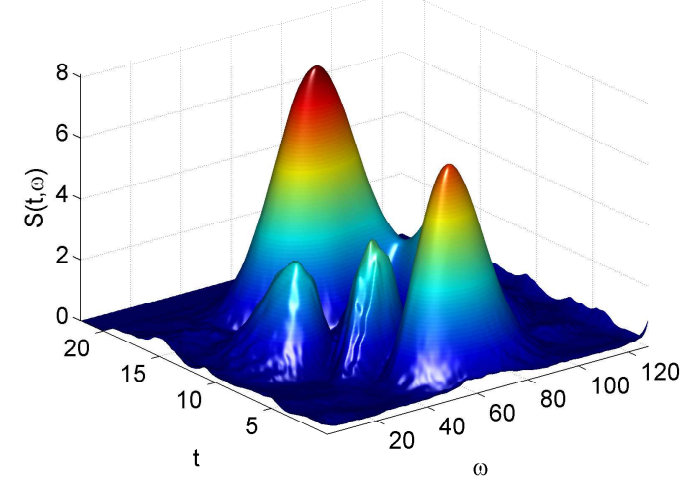

(b) Estimated ES

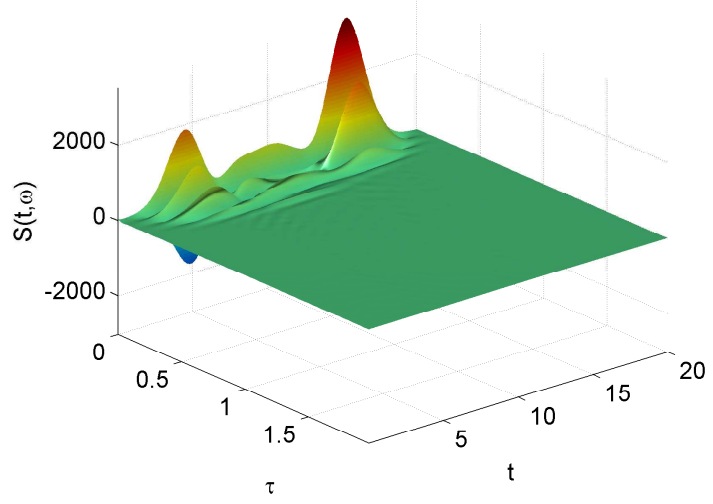

(d) Estimated ACF

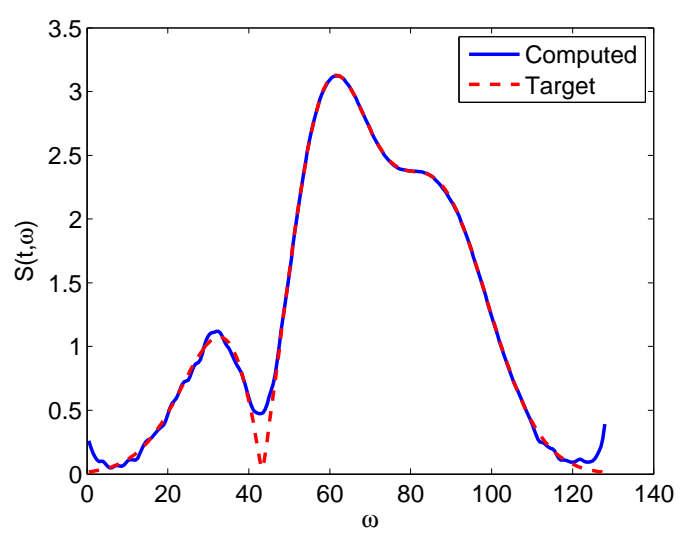

(f) Sample ES slice

Figure 15: MATLAB peaks numerical example results. After 200,000 iterations of the MGM, final error: $4.58 \%$ in the $\mathrm{ES}$ and $0.68 \%$ in the $\mathrm{ACF}$ 


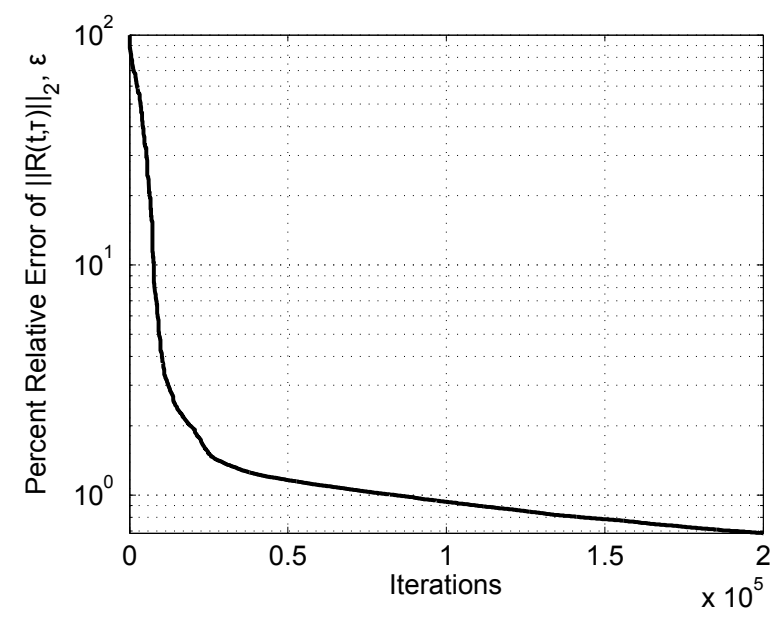

Figure 16: MGM MATLAB peaks example errors

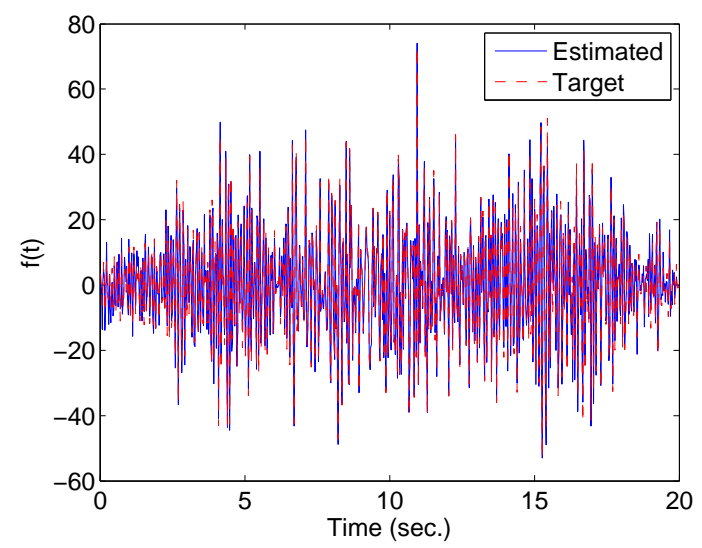

(a) Simulated sample realization comparison
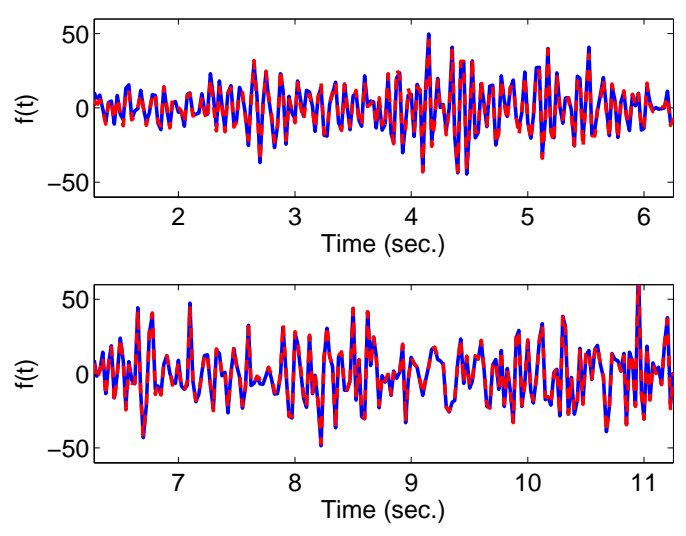

(b) Zoom in on sample realizations

Figure 17: Comparison of sample realizations generated using SRM with target ES (red dash) and estimated ES (solid blue) for peaks example 
Table 1: PM and MGM efficiency comparison

\begin{tabular}{|c|c|c|c|c|}
\hline & \multicolumn{2}{|c|}{ PM } & \multicolumn{2}{c|}{ MGM } \\
\hline & Iteration & $1,000,000$ iter. & Iteration & 130,000 iter. \\
\hline Fortran serial & $10.9870 \mathrm{sec}$ & 18 weeks & $11.3329 \mathrm{sec}$ & 2.4 weeks \\
\hline Fortran 24 core & $1.2887 \mathrm{sec}$ & 2 weeks & $1.6386 \mathrm{sec}$ & 2.5 days \\
\hline
\end{tabular}

each time instant is computed independently.

\section{Selection of initial evolutionary spectrum}

The perturbation method and the multi-grid method were developed to accept an arbitrary initial evolutionary spectrum. In the examples shown, a flat spectrum of uniform white noise was chosen with $A(t, \omega)=50 \forall t, \omega$. This initial evolutionary spectrum demonstrates the convergence from an arbitrary and unbiased initial spectrum making the case for existence and uniqueness. However, convergence from such a spectrum occurs very slowly and requires hundreds of thousands of iterations. If the initial evolutionary spectrum can be selected appropriately, then the number of iterations (and the computational cost) can be significantly reduced. The following section introduces a new quantity referred to as the "pseudo-spectrum" that is proposed for use as the initial guess for the spectrum. Some properties of the pseudo-spectrum are discussed along with its relation to the true evolutionary spectrum. Two examples are then considered to demonstrate the improvement in computational efficiency associated with using the pseudo-spectrum as an initial guess of the evolutionary spectrum.

\subsection{Definition of "Pseudo-Spectrum"}

Priestley's theory of evolutionary power is valid for the class of non-stationary stochastic processes that are referred to as "semi-stationary." A semi-stationary process is one whose modulating function given by Eq. (5) is slowly varying. The modulating function is considered slowly varying if its Fourier transform $d H(\omega, \theta)$ is highly concentrated in the region near $\omega=0$. A length-scale $B_{\mathcal{F}}(\omega)$ is defined for $d H(\omega, \theta)$ as:

$$
B_{\mathcal{F}}(\omega)=\int_{-\infty}^{\infty}|\theta||d H(\omega, \theta)|
$$


that is dependent on the specific family of functions used to characterize the modulating function and can be computed as described by Conte and Peng [51]. The process is said to be semi-stationary if $B_{\mathcal{F}}(\omega)<\infty, \forall \omega$. The semi-stationary process has a (functional family specific) characteristic width defined by:

$$
B_{\mathcal{F}}=\frac{1}{\sup _{\omega}\left(B_{\mathcal{F}}(\omega)\right)}
$$

and the absolute characteristic width considers all possible families of functions admitting the representation in Eq. (6) as:

$$
B_{\mathcal{X}}=\sup _{\mathcal{F}}\left(B_{\mathcal{F}}\right)
$$

The characteristic width is, generally speaking, a measure of the maximum interval over which the process may be considered approximately stationary.

Consider next the semi-stationary process with modulating function $A(t, \omega)$ selected according to the family of amplitude modulated complex exponentials as given in Eq. (4). The approximation $A(s, \omega) \approx A(t, \omega)$ is valid within the window $|s-t|<B_{\mathcal{F}}$. Under these conditions, Eq. (10) can be rewritten:

$$
R(t, \tau)=\int_{-\infty}^{+\infty} A^{2}(t, \omega) e^{i \omega \tau} \mathrm{d} \omega=\int_{-\infty}^{+\infty} \tilde{S}(t, \omega) e^{i \omega \tau} \mathrm{d} \omega
$$

For a fixed $t$, this expression is invertible as:

$$
\tilde{S}(t, \omega)=\frac{1}{2 \pi} \int_{-\infty}^{\infty} R(t, \tau) e^{-i \omega \tau} \mathrm{d} \tau
$$

The quantity $\tilde{S}(t, \omega)$ is referred to as the "pseudo-spectrum." It meets all of the requirements of a valid evolutionary spectrum for semi-stationary processes. In fact, for semi-stationary processes with strong correlation only in the window $\tau<B_{\mathcal{F}}$, the pseudo-spectrum will provide a near exact estimate of the true evolutionary spectrum. For those processes with appreciable correlation for $\tau>B_{\mathcal{F}}$, some noticeable error will exist in the pseudo-spectrum estimate of the evolutionary spectrum.

In the calculation of the pseudo-spectrum, it is important to note that $R(t, \tau)$ is defined in terms of a lag $\tau$ that is "two-sided" unlike the traditional "one-sided" lag for a stationary 
autocorrelation function. That is, $R(t, \tau) \neq R(t,-\tau)$ in general. Consequently, both positive and negative values of $\tau$ must be considered when computing the pseudo-spectrum according to Eq. (29). Furthermore, the user should make note of the discretization to avoid aliasing effects. Namely, the following requirements must be met:

$$
\begin{aligned}
T & =\frac{2 \pi}{\Delta \omega} \\
\Delta t & \leq \frac{2 \pi}{2 \omega_{u}}
\end{aligned}
$$

The pseudo-spectrum provides an initial estimate of the evolutionary spectrum - the accuracy of which is dependent on the relative scale of the characteristic width and the correlation length of the process. For this reason, it is used as a starting point for the MGM. The following examples demonstrate its effectiveness.

\subsection{Numerical examples}

In this section, two numerical examples are carried out with the MGM using the newly defined pseudo-spectrum as the initial guess of the evolutionary spectrum. Both examples are carried out on a $1024 \times 1024$ discretization in time and frequency.

\subsubsection{Clough-Penzien example}

Consider again the Clough-Penzien ES from Eqn. (18). The target ES is shown in Figure 18(a). The computed pseudo-spectrum using Eqn. (29) is shown in Figure 18(b). The error of this initial guess is $0.89 \%$ in the ACF and $2.55 \%$ in the ES, both of which fall below the error values of the PM and approach the error in correlation of the MGM. This is expected given that $B_{\mathcal{F}}=0.26 \mathrm{sec}$ while the range of appreciable correlation is less than about $0.3 \mathrm{sec}$ From Figure 18(b), it can be seen that there is some difficulty capturing $S(t, \omega=0)$. Sample slices of the pseudo-spectrum are also shown in Figure 18. Simulated sample realizations are shown in Figure 19. Figure 19(a) shows the entire duration and Figure 19(b) shows two five second windows. The simulated samples agree nicely, though some extra high frequency content can be noticed in the tail (between 15 and 20 seconds) as also observed by the

noisiness of the pseudo-spectrum shown in Figure 18(e). Still, these results show that the pseudo-spectrum is, in fact, a very good approximation of this ES. 


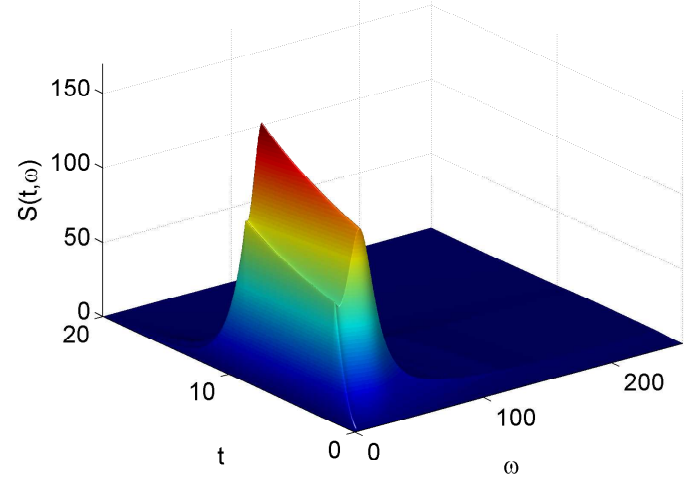

(a) Target Clough-Penzien ES

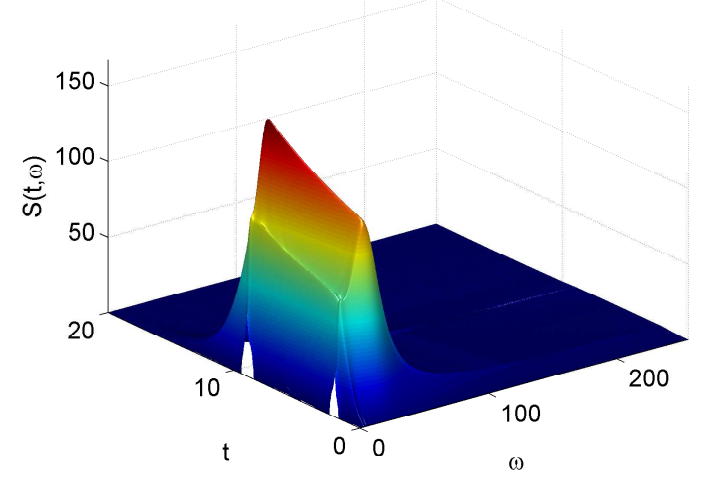

(b) Estimated pseudo-spectrum

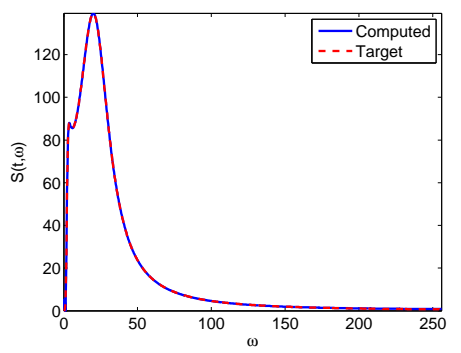

(c) $t=5 \mathrm{sec}$

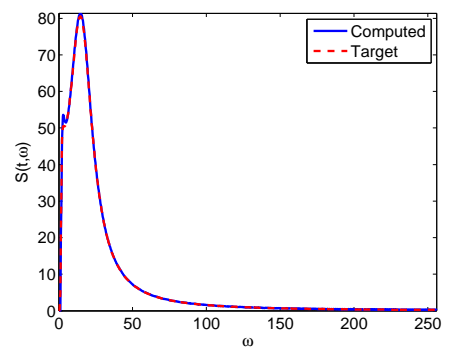

(d) $t=10 \mathrm{sec}$

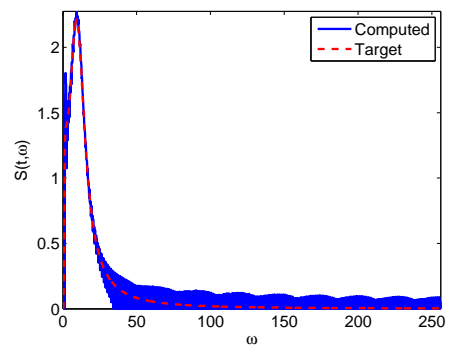

(e) $t=15 \mathrm{sec}$

Figure 18: Pseudo-spectrum results for Clough-Penzien example

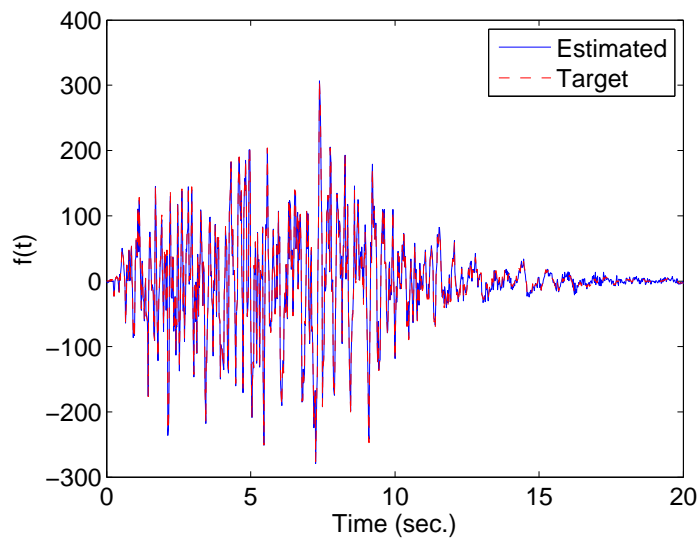

(a) Simulated sample realization comparison
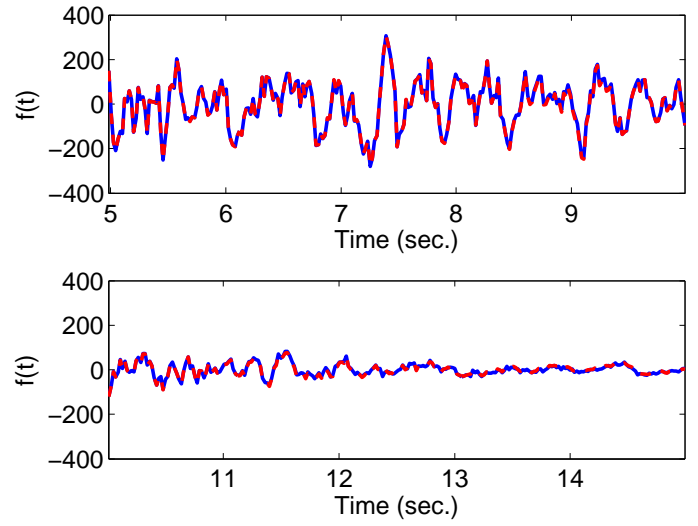

(b) Zoom in on sample realizations

Figure 19: Comparison of sample realizations generated using SRM with target ES (red dash) and estimated ES (solid blue) for pseudo-spectrum of Clough-Penzien example 


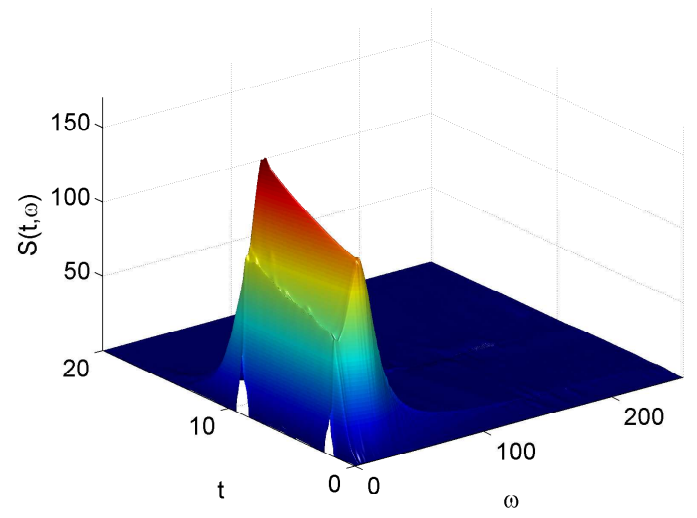

(a) Computed ES

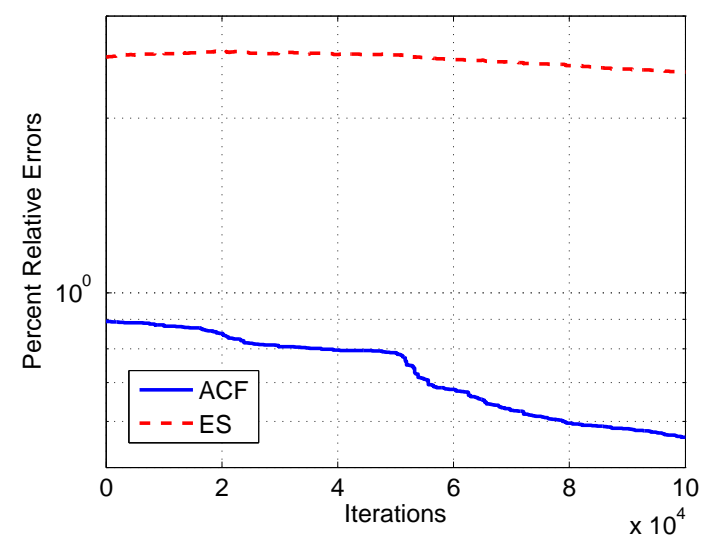

(b) Error vs. iterations

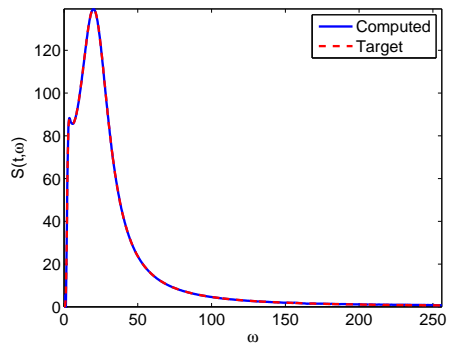

(c) $t=5 \mathrm{sec}$

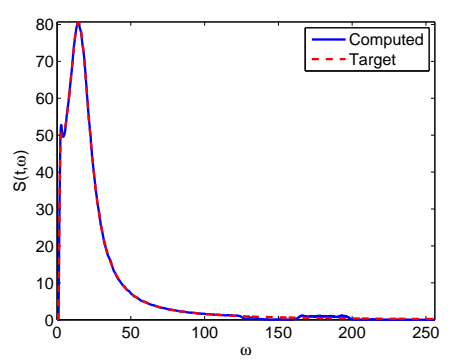

(d) $t=10 \mathrm{sec}$

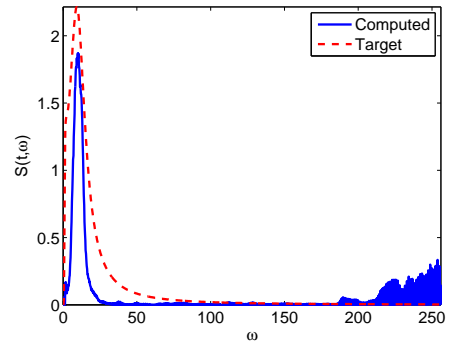

(e) $t=15 \mathrm{sec}$

Figure 20: MGM with pre-processing for Clough-Penzien example. Final errors after 100,000 iterations: $2.4 \%$ in $\mathrm{ES}$ and $0.56 \%$ in $\mathrm{ACF}$

In this case, it could be argued that the pseudo-spectrum is a sufficient approximation of the evolutionary spectrum for practical purposes and no perturbations are necessary. Nonetheless, the MGM was performed with the pseudo-spectrum of Figure 18(b) as an initial guess. The computed ES from the MGM iterations is shown in Figure 20(a), and the convergence is shown in Figure 20(b) for 100,000 iterations yielding $\epsilon=0.56 \%$ in the $\mathrm{ACF}$. The previously obtained error $\epsilon=0.64 \%$ has been achieved in approximately 70,000 iterations; a further reduction of nearly an order of magnitude. The final errors of $0.56 \%$ in the $\mathrm{ACF}$ and $2.4 \%$ in the ES are a significant improvement over the MGM with arbitrary initial spectrum largely because the boundary issues are alleviated. 


\subsubsection{Linear chirp process}

The final example considers the so-called linear chirp process, in which the central frequency of a narrow-band process changes linearly with time. This example possesses a high degree of non-stationarity if the central frequency changes rapidly testing the limits of the semi-stationary condition. The general chirp ES is defined as:

$$
S(t, \omega)=\exp \left[\frac{-\left(\omega-\omega_{0}(t)\right)^{2}}{4}\right]
$$

where, for the case of a linear chirp, the frequency shift parameter, $\omega_{0}(t)$ is defined as:

$$
\omega_{0}(t)=\frac{\alpha_{T}-\alpha_{0}}{T} t+\alpha_{0}
$$

where $\alpha_{0}$ is the value of $\omega_{0}(t=0), \alpha_{T}=\omega_{0}(t=T)$, and $T$ is the duration of the process . In this example, the following values are used:

$$
\begin{aligned}
\alpha_{0} & =\frac{\omega_{u}}{4} \\
\alpha_{T} & =\frac{3 \omega_{u}}{4} \\
\therefore \omega_{0}(t) & =\frac{\omega_{u}}{4}\left(2 \frac{t}{T}+1\right) \\
\omega_{u}=32 \mathrm{rad} / \mathrm{sec} &
\end{aligned}
$$

The target spectrum is shown in Figure 21(a) with the estimated pseudo-spectrum shown in Figure 21(b) and time slices comparing them in Figures 21(c) - 21(e). There are clearly edge effects near $t=0$ and $T$. Aside from these, the approximate shape is found, including the location of the peak, but with significant "blunting" of the peak. This is to be expected given that $B_{\mathcal{F}}=0.2 \mathrm{sec}$ and the extents of significant correlation are $\pm 3.2 \mathrm{sec}$ as shown in Figure 22. This pseudo-spectrum produces an error of $11.18 \%$ in the ACF and $19.67 \%$ in the ES. Consequently, the MGM method should be used to improve the estimate. Thus, for the linear chirp process, there seems to still be a clear call for perturbations.

The computed ES from the MGM iterations with pseudo-spectrum initial evolutionary spectrum is shown in Figure 23(a), and the convergence is shown in Figure 23(b) for 100,000 


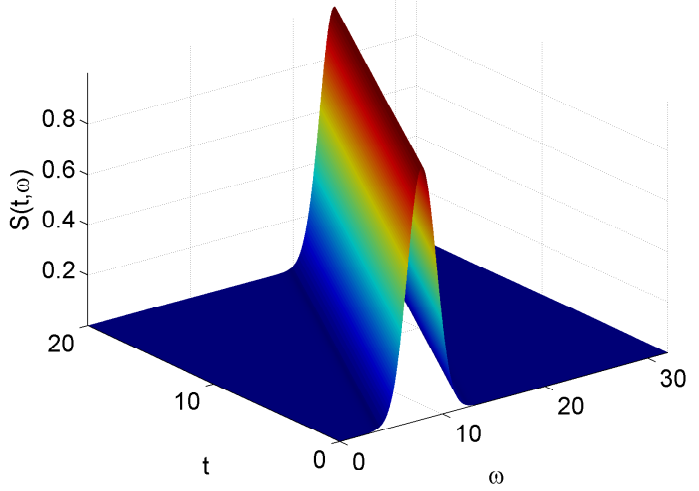

(a) Target linear chirp ES

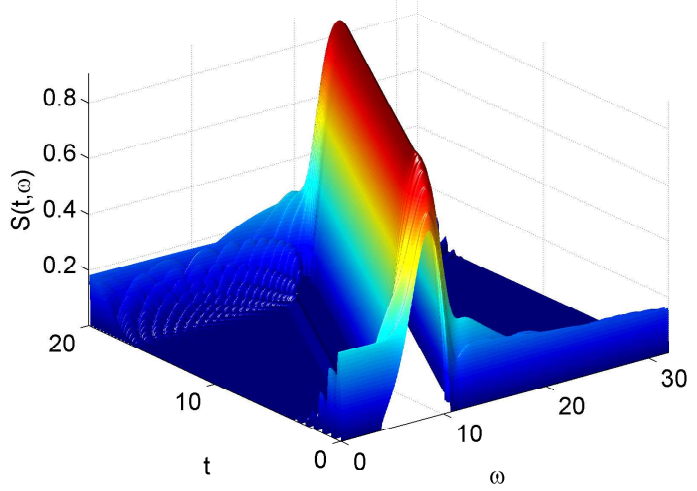

(b) Estimated pseudo-spectrum

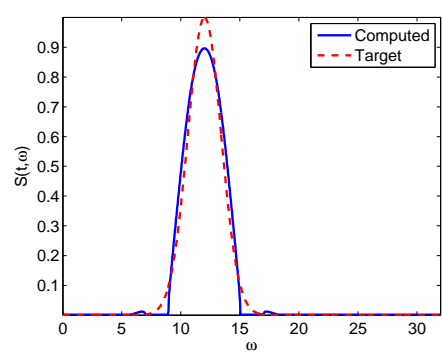

(c) $t=5 \mathrm{sec}$

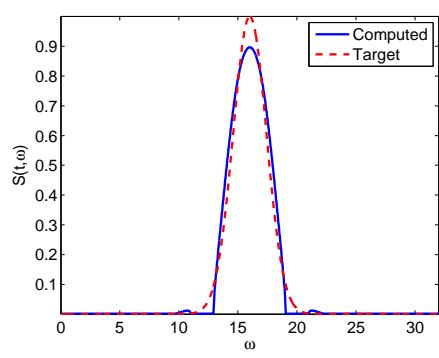

(d) $t=10 \mathrm{sec}$

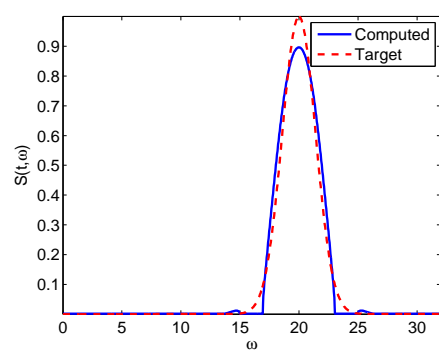

(e) $t=15 \mathrm{sec}$

Figure 21: Pseudo-spectrum results for linear chirp example

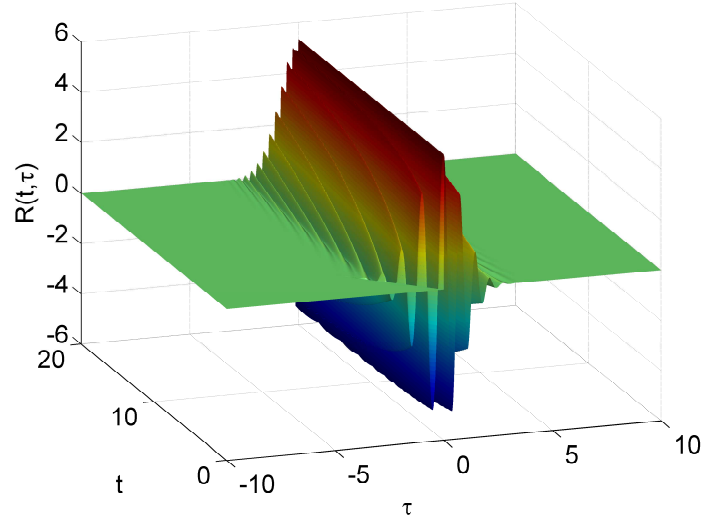

(a) Surface plot

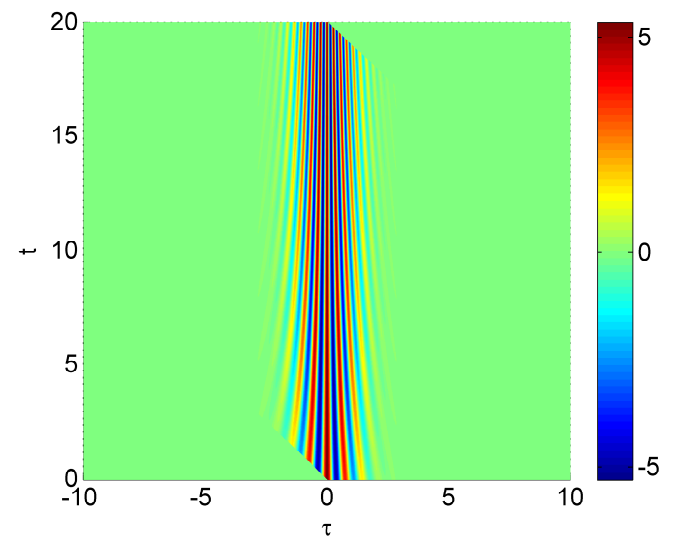

(b) Color map

Figure 22: Auto-correlation function for linear chirp example 


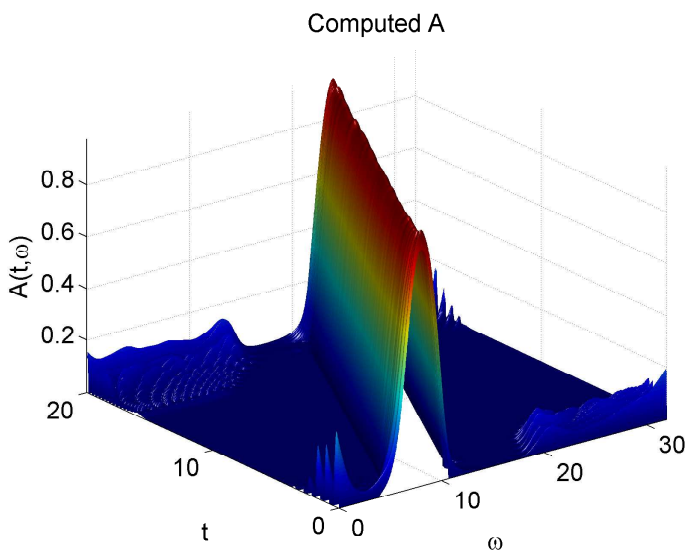

(a) Computed ES

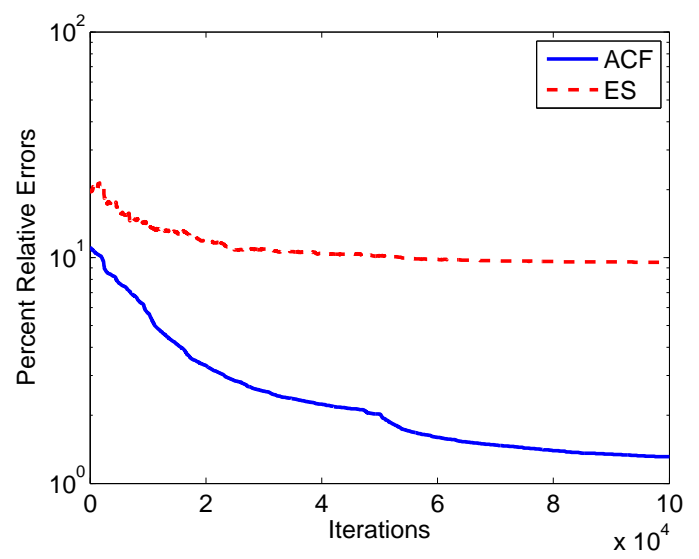

(b) Error vs. iterations

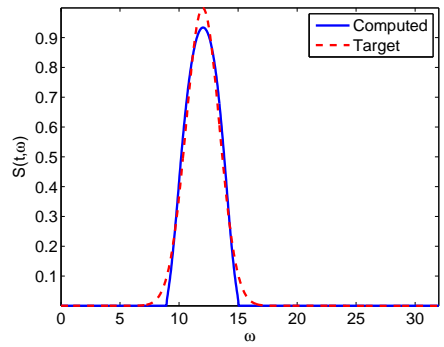

(c) $t=5 \mathrm{sec}$

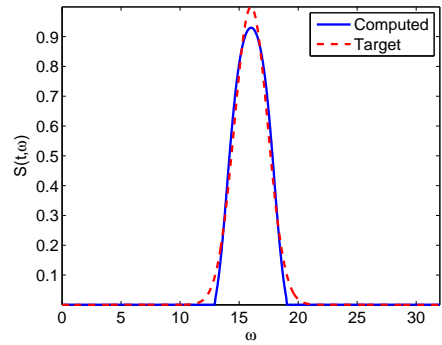

(d) $t=10 \mathrm{sec}$

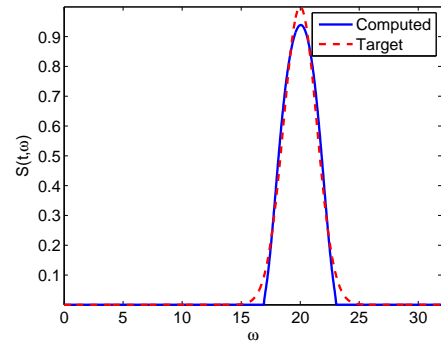

(e) $t=15 \mathrm{sec}$

Figure 23: MGM with pre-processing for linear chirp example. Final errors after 100,000 iterations: 9.52\% in $\mathrm{ES}$ and $1.31 \%$ in $\mathrm{ACF}$

iterations with final errors of $1.3 \%$ in the ACF and $9.5 \%$ in the ES. The ACF error has been reduced drastically, and the ES error has been improved as well. Note that the majority of the remaining error in the evolutionary spectrum is on the boundaries and could potentially be cleaned up in post-processing or imposing additional constraints on the MGM optimization. Simulated sample realizations are shown in Figure 24. Figure 24(a) shows the entire duration and Figure 24(b) shows two five second window zoom-ins. Overall, there is good agreement between the sample realizations.

\section{Conclusions}

This work began by examining the existence and uniqueness of the evolutionary spectrum computed by inverting the expression established by Priestley [31] for computing the autocorrelation function from the evolutionary spectrum under specific conditions. No such 


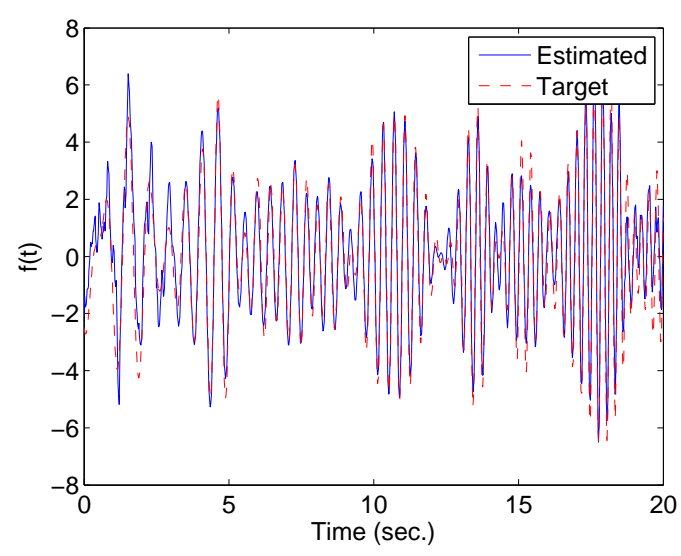

(a) Simulated sample realization comparison
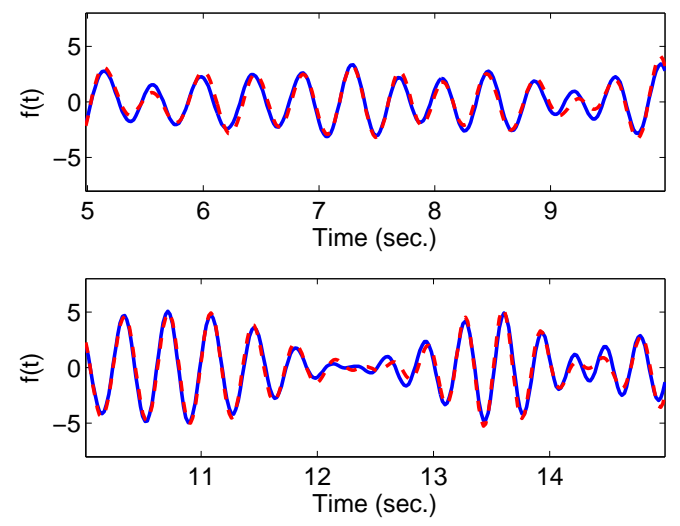

(b) Zoom in on sample realizations

Figure 24: Comparison of sample realizations generated using SRM with target ES (red dash) and estimated ES (solid blue) for MGM with pseudo-spectrum for linear chirp example

analytical inverse exists but the existence and uniqueness of such an inversion was shown to likely exist through a numerical optimization routine. This work represents the first such evidence that a unique inverse may exist. A methodology for computing this inverse as a constrained optimization problem was presented relying on acceptance/rejection of random perturbations to the estimated evolutionary spectrum. The method is computationally very expensive. To improve the efficiency, a Multi-Grid Method inspired by the multi-grid solvers for linear systems is proposed and coupled with a newly defined "pseudo-spectrum" for the initial estimation of the evolutionary spectrum. These developments afford reduction in computational cost by two orders of magnitude or more. The ability to estimate the evolutionary spectrum from a non-stationary autocorrelation function represents an important development for the simulation of non-stationary stochastic processes and especially those possessing strongly non-Gaussian marginal PDFs.

\section{Acknowledgments}

This work was supported in part by the National Science Foundation under IGERT Award \#DGE-0905597, Solving Urbanization Challenges By Design. 


\section{References}

[1] M. Shinozuka, C. Jan, Digital simulation of random processes and its applications, Journal of Sound and Vibrationon 25 (1) (1972) 111-128.

[2] M. Shields, G. Deodatis, P. Bocchini, A simple and efficient methodology to approximate a general non-Gaussian stationary stochastic process by a translation process, Probabilistic Engineering Mechanics 26 (4) (2011) 511-519.

[3] M. Shields, G. Deodatis, A simple and efficient methodology to approximate a general non-gaussian stationary stochastic vector process by a translation process with applications in wind velocity simulation, Probabilistic Engineering Mechanics 31 (2013) 19-29.

[4] M. D. Shields, G. Deodatis, Estimation of evolutionary spectra for simulation of nonstationary and non-Gaussian stochastic processes, Computers \& Structures 126 (2013) $149-163$.

[5] M. Grigoriu, Applied non-Gaussian processes: examples, theory, simulation, linear random vibration, and MATLAB solutions, PTR Prentice Hall, Englewood Cliffs, N.J., 1995.

[6] W. Gersch, J. Yonemoto, Synthesis of multivariate random vibration systems: a twostage least squares AR-MA model approach, Journal of Sound and Vibration 52 (4) (1977) 553-565.

[7] P. Spanos, J. Hansen, Linear prediction theory for digital simulation of sea waves, Journal of Energy Resources Technology 103 (1981) 243-249.

[8] F. Kozin, F. Nakajima, The order determination problem for linear time-varying AR models, IEEE Transactions on Automatic Control AC-25 (2) (1980) 250-257.

[9] P. Spanos, ARMA algorithms for ocean wave modeling, Journal of Energy Resources Technology 105 (1983) 300-309. 
[10] P. Spanos, G. Solomos, Markov approximation to transient vibration, Journal of Engineering Mechanics 109 (4) (1983) 1134-1150.

[11] E. Samaras, M. Shinzuka, A. Tsurui, ARMA representation of random processes, Journal of Engineering Mechanics 111 (3) (1985) 449-461.

[12] K. A. R. I. Karhunen, Uber lineare Methoden in dew Wahrscheinlichkeitsrechnung, Ann. Acad. Sci. Fennicae. 37 (1947) 3-79.

[13] M. Kac, A. Siegert, An explicit representation of a stationary Gaussian process, The Annals of Mathematical Statistics 18 (3) (1947) 438-442.

[14] M. Loeve, Fonctions aléatoires du second ordre, A note in P, Levi's Processus stochastiques et mouvement Brownien, Gauthier-Villars, Paris.

[15] R. G. Ghanem, P. D. Spanos, Stochastic Finite Elements: A Spectral Approach, revised Edition, Dover, 2012.

[16] Pacific-Earthquake-Engineering-Research-Center, PEER Ground Motion Database (2013).

[17] S. Amada, T. Munekata, Y. Nagase, Y. Ichikawa, A. Kirigai, Y. Zhifei, The Mechanical Structures of Bamboos in Viewpoint of Functionally Gradient and Composite Materials, Journal of Composite Materials 30 (7) (1996) 800-819.

[18] S. Amada, Y. Ichikawa, T. Munekata, Y. Nagase, H. Shimizu, Fiber texture and mechanical graded structure of bamboo, Composites Part B: Engineering 28 (1-2) (1997) $13-20$.

[19] C. H. Page, Instantaneous Power Spectra, Journal of Applied Physics 23 (1) (1952) $103-106$.

[20] W. Martin, P. Flandrin, Wigner-Ville spectral analysis of nonstationary processes, IEEE Transactions on Acoustics, Speech, and Signal Processing 33 (6) (1985) 1461-1470. 
[21] J. Hammond, R. Harrison, Wigner-Ville and evolutionary spectra for covariance equivalent nonstationary random processes, IEEE International Conference on Acoustics, Speech, and Signal Processing 10 (1985) 1025-1028.

[22] J. Hammond, J. Lee, R. Harrison, The relationship between Wigner-Ville and evolutionary spectra for frequency modulated random processes, in: IEEE International Conference on Acoustics, Speech, and Signal Processing, Vol. 11, 1986, pp. 2327-2330.

[23] M. B. Priestley, Wavelets and time-dependent spectral analysis, Journal of Time Series Analysis 17 (1) (1996) 85-103.

[24] R. von Sachs, K. Schneider, Wavelet smoothing of evolutionary spectra by nonlinear thresholding, Applied and Computational Harmonic Analysis 3 (1996) 268-282.

[25] M. H. Neumann, R. von Sachs, Wavelet thresholding in anisotropic function classes and application to adaptive estimation of evolutionary spectra, The Annals of Statistics 25 (1) (1997) 38-76.

[26] K. Gurley, A. Kareem, Applications of wavelet transforms in earthquake, wind and ocean engineering, Engineering Structures (1999) 149-167.

[27] G. P. Nason, R. von Sachs, G. Kroisandt, Wavelet processes and adaptive estimation of the evolutionary wavelet spectrum, Journal of the Royal Statistical Society. Series B (Statistical Methodology) 62 (2) (2000) 271-292.

[28] P. D. Spanos, G. Failla, Evolutionary spectra estimation using wavelets, Journal of Engineering Mechanics 130 (2004) 952-960.

[29] P. D. Spanos, J. Tezcan, P. Tratskas, Stochastic processes evolutionary spectrum estimation via harmonic wavelets, Computer Methods in Applied Mechanics and Engineering 194 (12-16) (2005) 1367-1383. 
[30] P. Spanos, I. Kougioumtzoglou, Harmonic wavelets based statistical linearization for response evolutionary power spectrum determination, Probablistic Engineering Mechanics 27 (2012) 57-68.

[31] M. Priestley, Non-linear and Non-stationary Time Series Analysis, Academic Press, 1988.

[32] M. B. Priestley, Evolutionary spectra and non-stationary processes, Journal of the Royal Statistical Society. Series B (Statistical Methodology) 27 (2) (1965) 204-237.

[33] D. Schillinger, V. Papadopoulos, Accurate estimation of evolutionary power spectra for strongly narrow-band random fields, Computer Methods in Applied Mechanics and Engineering 199 (2010) 947-960.

[34] M. D. Shields, Simulation of Stochastic Processes: Applications in Civil Engineering, Ph.D. thesis, Columbia University, New York, NY (2010).

[35] M. Shields, Simulation of spatially correlated nonstationary response spectrumcompatible ground motion time histories, Journal of Engineering Mechanics 141 (6) (2015) 04014161.

[36] S. Kirkpatrick, C. Gelatt, M. Vecchi, Optimization by simulated annealing, Science 220 (1983) 671-680.

[37] V. Cerny, A thermodynamical approach to the traveling salesman problem: An efficient simulation algorithm, Tech. rep., Comenius University (1982).

[38] V. Cerny, Thermodynamical approach to the traveling salesman problem: an efficient simulation algorithm, Journal of Optimization Theory and Applications 45 (1985) 4151.

[39] P. Laarhoven, E. Aarts, Simulated annealing: Theory and applications, Kluwer Academic Publishers, D. Reidel Publishing Company, Dordrecht, The Netherlands, 1987. 
[40] G. Deodatis, Non-stationary stochastic vector processes: seismic ground motion applications, Probabilistic Engineering Mechanics 11 (3) (1996) 149-167.

[41] R. Clough, J. Penzien, Dynamics of Structures, McGraw Hill, 1975.

[42] G. Deodatis, M. Shinozuka, Auto-regressive model for nonstationary stochastic processes, Journal of Engineering Mechanics 114 (11) (1988) 1995-2012.

[43] P. Jennings, G. Housner, N. Tsai, Simulated earthquake motions, Tech. rep., Earthquake Engineering Research Laboratory, California Institute of Technology, Pasadena, California (1968).

[44] K. Kanai, Seismic-empirical formula for the seismic characteristics of the ground, Bulletin of Earthquake Research Institute 35 (1957) 309-325.

[45] H. Tajimi, A statistical method of determining the maximum response of a building structure during an earthquake, in: Proceedings of the 2nd world conference on earthquake engineering, 1960, pp. 781-798.

[46] B. R. Ellingwood, M. E. Batts, Characterization of earthquake forces for probabilitybased design of nuclear structures, Tech. rep., Center for Building Technology, National Bureau of Standards, Washington, DC (USA) (1982).

[47] A. Brandt, Multi-level adaptive solutions to boundary-value problems, Mathematics of Computation 31 (138) (1977) 333-390.

[48] W. L. Briggs, V. E. Henson, S. F. McCormick, A Multigrid Tutorial, 2nd Edition, SIAM, 2000.

[49] MATLAB, version 8.0.0.783 (R2012b), The MathWorks Inc., Natick, Massachusetts, 2012.

[50] C. Yeong, S. Torquato, Reconstructing random media, Physical Review E: Statistical, nonlinear, and soft matter physics 57 (1998) 495-506. 
[51] J. Conte, B. Peng, Fully nonstationary analytical earthquake ground-motion model, Journal of Engineering Mechanics 123 (1997) 15-24. 